\title{
Abundances of sulphur molecules in the Horsehead nebula
}

\section{First $\mathrm{NS}^{+}$detection in a photodissociation region}

\author{
P. Rivière-Marichalar ${ }^{1,2}$, A. Fuente ${ }^{2}$, J. R. Goicoechea ${ }^{1}$, J. Pety ${ }^{3,4}$, R. Le Gal ${ }^{5}$, P. Gratier ${ }^{6}$, \\ V. Guzmán ${ }^{7}$, E. Roueff ${ }^{8}$, J. C. Loison ${ }^{9}$, V. Wakelam ${ }^{6}$, and M. Gerin ${ }^{4}$ \\ ${ }^{1}$ Instituto de Física Fundamental (CSIC), Calle Serrano 121, 28006 Madrid, Spain \\ 2 Observatorio Astronómico Nacional (OAN,IGN), Apdo 112, 28803 Alcalá de Henares, Spain \\ e-mail: p.riviere@oan.es \\ ${ }^{3}$ Institut de Radioastronomie Millimétrique (IRAM), 300 rue de la Piscine, 38406 Saint Martin d'Hères, France \\ ${ }^{4}$ LERMA, Observatoire de Paris, PSL Research University, CNRS, Sorbonne Universités, UPMC Université Paris 06, \\ Ecole Normale Supérieure, 75005 Paris, France \\ ${ }^{5}$ Harvard-Smithsonian Center for Astrophysics, 60 Garden St., Cambridge, MA 02138, USA \\ ${ }^{6}$ Laboratoire d'Astrophysique de Bordeaux, University of Bordeaux, CNRS, B18N, allée Geoffroy Saint-Hilaire, \\ 33615 Pessac, France \\ ${ }^{7}$ Instituto de Astrofísica, Pontificia Universidad Católica de Chile, Av. Vicuña Mackenna, 4860, 7820436 Macul, Santiago, Chile \\ ${ }^{8}$ LERMA, Observatoire de Paris, PSL Research University, CNRS, Sorbonne Universités, UPMC Université Paris 06, \\ 92190 Meudon, France \\ ${ }^{9}$ Institut des Sciences Moléculaires de Bordeaux (ISM), CNRS, University of Bordeaux, 351 cours de la Libération, \\ 33400 Talence, France
}

Received 25 February 2019 / Accepted 3 June 2019

\begin{abstract}
Context. Sulphur is one of the most abundant elements in the Universe $\left(\mathrm{S} / \mathrm{H} \sim 1.3 \times 10^{-5}\right)$ and plays a crucial role in biological systems on Earth. The understanding of its chemistry is therefore of major importance.

Aims. Our goal is to complete the inventory of S-bearing molecules and their abundances in the prototypical photodissociation region (PDR) the Horsehead nebula to gain insight into sulphur chemistry in UV irradiated regions. Based on the WHISPER (Wide-band High-resolution Iram-30 m Surveys at two positions with Emir Receivers) millimeter (mm) line survey, our goal is to provide an improved and more accurate description of sulphur species and their abundances towards the core and PDR positions in the Horsehead. Methods. The Monte Carlo Markov chain (MCMC) methodology and the molecular excitation and radiative transfer code RADEX were used to explore the parameter space and determine physical conditions and beam-averaged molecular abundances.

Results. A total of $13 \mathrm{~S}$-bearing species (CS, SO, SO $2, \mathrm{OCS}, \mathrm{H}_{2} \mathrm{CS}-$ both ortho and para - $\mathrm{HDCS}, \mathrm{C}_{2} \mathrm{~S}, \mathrm{HCS}^{+}, \mathrm{SO}^{+}, \mathrm{H}_{2} \mathrm{~S}, \mathrm{~S}_{2} \mathrm{H}, \mathrm{NS}$ and $\mathrm{NS}^{+}$) have been detected in the two targeted positions. This is the first detection of $\mathrm{SO}^{+}$in the Horsehead and the first detection of $\mathrm{NS}^{+}$in any PDR. We find a differentiated chemical behaviour between $\mathrm{C}-\mathrm{S}$ and $\mathrm{O}-\mathrm{S}$ bearing species within the nebula. The $\mathrm{C}-\mathrm{S}$ bearing species $\mathrm{C}_{2} \mathrm{~S}$ and $\mathrm{o}-\mathrm{H}_{2} \mathrm{CS}$ present fractional abundances a factor of $>$ two higher in the core than in the PDR. In contrast, the $\mathrm{O}-\mathrm{S}$ bearing molecules $\mathrm{SO}, \mathrm{SO}_{2}$, and OCS present similar abundances towards both positions. A few molecules, $\mathrm{SO}^{+}, \mathrm{NS}$, and $\mathrm{NS}^{+}$, are more abundant towards the PDR than towards the core, and could be considered as PDR tracers.

Conclusions. This is the first complete study of S-bearing species towards a PDR. Our study shows that CS, $\mathrm{SO}$, and $\mathrm{H}_{2} \mathrm{~S}$ are the most abundant S-bearing molecules in the PDR with abundances of approximately a few $10^{-9}$. We recall that $\mathrm{SH}, \mathrm{SH}^{+}, \mathrm{S}$, and $\mathrm{S}^{+}$are not observable at the wavelengths covered by the WHISPER survey. At the spatial scale of our observations, the total abundance of $\mathrm{S}$ atoms locked in the detected species is $<10^{-8}$, only $\sim 0.1 \%$ of the cosmic sulphur abundance.
\end{abstract}

Key words. astrochemistry - ISM: abundances - ISM: kinematics and dynamics - ISM: molecules - stars: formation stars: low-mass

\section{Introduction}

Sulphur is one of the most abundant elements in the Universe $\left(\mathrm{S} / \mathrm{H} \sim 1.3 \times 10^{-5}\right.$ in the solar photosphere, Asplund et al. 2005) and plays a crucial role in biological systems on Earth. Surprisingly, sulphuretted molecules are not as abundant as expected in the interstellar medium. A few sulphur compounds have been detected in diffuse clouds demonstrating that the sulphur abundance in these low density regions is close to the cosmic value (Neufeld et al. 2015). A moderate sulphur depletion (a factor of four) is estimated in the external layers of the photodissociation region (PDR) in the Horsehead nebula (Goicoechea et al. 2006). In cold molecular clouds, a large depletion of sulphur is usually considered to reproduce the observations (see for instance Tieftrunk et al. 1994; Vastel et al. 2018). The depletion of sulphur is observed not only in cold pre-stellar cores, but also in hot cores/corinos (Wakelam et al. 2004). One would expect that most of the sulphur is locked in the icy grain mantles in dense cores but we should see almost all sulphur return to the gas phase in hot cores and strong shocks. However, even in the well-known Orion-KL hot core where the icy grain mantles are expected to evaporate releasing the molecules to the gas phase, one needs to assume a sulphur depletion of a factor of approximately ten to reproduce the observations (Esplugues et al. 2014; Crockett et al. 2014). Because of the high hydrogen abundances and the mobility of hydrogen in the ice matrix, sulphur atoms impinging on interstellar ice mantles are expected to form $\mathrm{H}_{2} \mathrm{~S}$ preferentially. However, 
there are only upper limits of the solid $\mathrm{H}_{2} \mathrm{~S}$ abundance (e.g. Jiménez-Escobar \& Muñoz Caro 2011). Oba et al. (2018) show that, at high densities, chemical desorption is likely to exceed photodesorption, thus becoming an efficient way of removing $\mathrm{H}_{2} \mathrm{~S}$ from the surface of grains.

Thus far, OCS is the only S-bearing molecule unambiguously detected in interstellar ice because of its large band strength in the infrared (Geballe et al. 1985; Palumbo et al. 1995); tentatively, $\mathrm{SO}_{2}$ has also been detected (Boogert et al. 1997). The bulk of the S-budget might be locked in atomic sulphur in the gas phase and/or in S-polymers in the solid phase, which are not easily observable (Jiménez-Escobar et al. 2014; Wakelam et al. 2004). The recent detection of the doubly-sulphuretted species $\mathrm{S}_{2} \mathrm{H}$ in the Horsehead nebula adds important information as well as new questions to the overall complex sulphur chemistry problem, possibly supporting the scenario of an important role of surface chemistry in the formation of the gaseous sulphuretted compounds (Fuente et al. 2017). Observations of the comet 67P with Rosetta showed that $\mathrm{H}_{2} \mathrm{~S}$ and atomic $\mathrm{S}$ are the most important $\mathrm{S}$-bearing species in cometary ices, with an abundance of $0.015 \%$ relative to water (Calmonte et al. 2016; Bockelée-Morvan \& Biver 2017).

Only a few PDRs have been investigated in detail taking advantage of their proximity and favourable edge-on geometry: NGC 7023 (Fuente et al. 1993, 1996, 2000), the Orion Bar (Cuadrado et al. 2015, 2017; Goicoechea et al. 2016, 2017), Mon R2 (Ginard et al. 2012; Treviño-Morales et al. 2014, 2016; Pilleri et al. 2012, 2013, 2014), and the Horsehead nebula. Here we investigate the sulphur chemistry in the Horsehead nebula. At a distance of $400 \mathrm{pc}$, the Horsehead is a PDR viewed nearly edge-on and illuminated by the $09.5 \mathrm{~V}$ star $\sigma$ Ori at a projected distance of $\sim 3.5 \mathrm{pc}$. The intensity of the incident far ultra-violet (FUV) radiation field is $\chi=60$ (Pety et al. 2005) relative to the interstellar radiation field in Draine units (Draine 1978). This PDR presents a differentiated chemistry from others associated with warmer regions such as the Orion Bar, NGC 7023, and Mon R2. One main difference is that the dust temperature is around $\sim 20-30 \mathrm{~K}$ in the PDR (Goicoechea et al. 2009), meaning it is below or close to the sublimation temperature of many species, allowing a rich surface chemistry on the irradiated surfaces as has been demonstrated by recent observational and theoretical studies (Guzmán et al. 2011, 2013). A first analysis of the sulphur chemistry was carried out by Goicoechea et al. (2006) who derived a gas-phase sulphur abundance of $\mathrm{S} / \mathrm{H}=(3.5 \pm 1.5) \times 10^{-6}$ in the low extinction PDR on the basis of single-dish and interferometric observations of the CS and $\mathrm{HCS}^{+}$ millimeter lines.

The Horsehead Wide-band High-resolution Iram-30 m Surveys at two positions with Emir Receivers (WHISPER) project is a complete unbiased line survey of the 3,2, and $1 \mathrm{~mm}$ bands using the Institut de Radioastronomie Millimétrique (IRAM) $30 \mathrm{~m}$ telescope. WHISPER has provided valuable hints regarding the chemistry of this region. The detection of the molecular ion $\mathrm{CF}^{+}$towards the $\mathrm{HCO}$ peak is well understood in terms of gasphase photochemistry (Guzmán et al. 2012). Photo-destruction of large polyatomic molecules or small grains into smaller hydrocarbon precursors is invoked to explain the extraordinarily high abundance of small hydrocarbons, such as $\mathrm{C}_{2} \mathrm{H}, \mathrm{C}_{3} \mathrm{H}_{2}, \mathrm{C}_{3} \mathrm{H}$, and $\mathrm{C}_{3} \mathrm{H}^{+}$(Pety et al. 2012; Guzmán et al. 2015). The detection of several complex organic molecules (COMs) towards the warm ( $\left.T_{\text {kin }} \sim 60 \mathrm{~K}\right)$ PDR was unexpected. In fact, the chemical complexity reached in the Horsehead is surprisingly high, with COMs of up to seven atoms: $\mathrm{HCOOH}, \mathrm{H}_{2} \mathrm{CCO}, \mathrm{CH}_{3} \mathrm{CHO}$, and $\mathrm{CH}_{3} \mathrm{CCH}$ (Guzmán et al. 2014). Current pure gas-phase models cannot reproduce the inferred $\mathrm{H}_{2} \mathrm{CO}, \mathrm{CH}_{3} \mathrm{OH}$, and COMs abundances in the Horsehead PDR (Guzmán et al. 2011, 2013), which supports the theory of a grain surface origin for these molecules. Le Gal et al. (2017) were able to reproduce the observed COMs abundances using a chemical model with grain surface chemistry and proposed that chemical desorption, instead of photodesorption, was most likely the dominant process to release COMs to the gas phase. However, the efficiency of chemical desorption seems to be extremely dependent on the species involved and the chemical composition of the icy mantle (Minissale et al. 2016; Oba et al. 2018). $\mathrm{CH}_{3} \mathrm{CN}$ and $\mathrm{CH}_{3} \mathrm{NC}$, key species for the formation of prebiotic molecules, seem to have a very specific formation pathway in this PDR (Gratier et al. 2013). In this paper we use the data of the Horsehead WHISPER project to investigate the chemistry of S-bearing species in this prototypical nebula.

\section{Observations and data reduction}

The data used in this work are taken from the Horsehead WHISPER (Wide-band High-resolution Iram-30 m Surveys at two positions with Emir Receivers, PI J. Pety) project. The Horsehead WHISPER project is a complete unbiased line survey of the 3,2 , and $1 \mathrm{~mm}$ bands using the IRAM $30 \mathrm{~m}$ telescope. Two positions were observed within this survey: (i) the PDR position, where HCO emission peaks $\left(\mathrm{RA}=5^{\mathrm{h}} 40^{\mathrm{m}} 53.936\right.$, Dec $\left.=02^{\circ} 28^{\prime} 00^{\prime \prime}, \mathrm{J} 2000\right)$ at the UV-illuminated surface of the Horsehead nebula (Gerin et al. 2009a, hereafter, PDR), and (ii) the core, where $\mathrm{DCO}^{+}$peaks $\left(\mathrm{RA}=5^{\mathrm{h}} 40^{\mathrm{m}} 55 \mathrm{~s} 61\right.$, Dec $=$ $\left.02^{\circ} 27^{\prime} 38^{\prime \prime}, \mathrm{J} 2000\right)$, which corresponds to a cold $\left(T_{\mathrm{k}} \sim 10-20 \mathrm{~K}\right.$, Pety et al. 2007) and UV-shielded condensation located less than $40^{\prime \prime}$ away from the PDR edge (hereafter, core). During the observations we used the position-switching procedure with the reference position located at an offset $\left(-100^{\prime \prime}, 0\right)$ relative to $\mathrm{RA}=05^{\mathrm{h}} 40^{\mathrm{m}} 54.27 \mathrm{Dec}=-02^{\circ} 28^{\prime} 00^{\prime \prime}$. 0 . In order to avoid relative calibration errors we integrated towards the $\mathrm{HCO}$ peak and the $\mathrm{DCO}^{+}$peak alternatively, changing from one position to the other every $15 \mathrm{~min}$. Line intensities are given in main brightness temperature $\left(T_{\mathrm{MB}}\right)$ and the frequency resolution in our survey is $49 \mathrm{kHz}$. The uncertainties in $T_{\mathrm{MB}}$ due to calibration errors are $\sim 10 \%$.

In Fig. 1, we show the integrated intensity maps of the ${ }^{34} \mathrm{SO} 2{ }_{3} \rightarrow 1_{2}, \mathrm{CCS} 8_{7} \rightarrow 7_{6} \mathrm{H}_{2} \mathrm{~S} 1_{1,0} \rightarrow 1_{0,1}$, and CS $2 \rightarrow 1$ lines as observed with the IRAM $30 \mathrm{~m}$ telescope. These maps were observed in previous projects by our team. The two targeted positions within WHISPER, PDR and core, are marked with filled squares and the 3 and $2 \mathrm{~mm}$ beam of the $30 \mathrm{~m}$ telescope are also shown. The $\mathrm{H}_{2} \mathrm{~S} 1_{1,0} \rightarrow 1_{0,1}$ line has been presented in Fuente et al. (2017). The other lines were observed in November and December 2017 under good winter weather conditions $(2 \mathrm{~mm}$ of precipitable water vapour) using the Eight MIxer Receiver (EMIR) sideband-separation receivers. The position-switching, on-the-fly observing mode was used, with off-position offsets $(\delta \mathrm{RA}, \delta \mathrm{Dec})=\left(-100^{\prime \prime}, 0^{\prime \prime}\right)$, that is, into the HII region. We observed along and perpendicular to the direction of the exciting star in zigzags, covering an area of $200^{\prime \prime} \times 200^{\prime \prime}$. The maps were processed with the Grenoble Image and Line Data Analysis System $\left(\right.$ GILDAS $^{1}$ ) software (Pety et al. 2005). The data were first calibrated to the $T_{\mathrm{a}}^{*}$ scale using the chopperwheel method (Penzias \& Burrus 1973), and then converted

1 See http://www.iram. fr/IRAMFR/GILDAS for more information about the GILDAS softwares. 
$\mathrm{T}_{\mathrm{mb}}(\mathrm{K})$

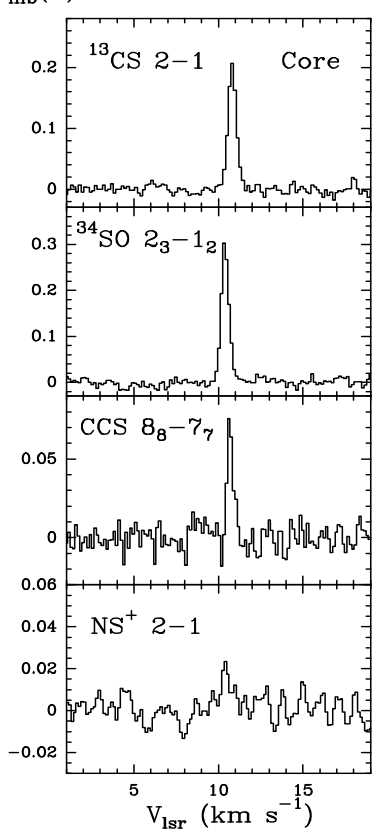

$\left(\mathrm{K} \mathrm{km} \mathrm{s}^{-1}\right)$

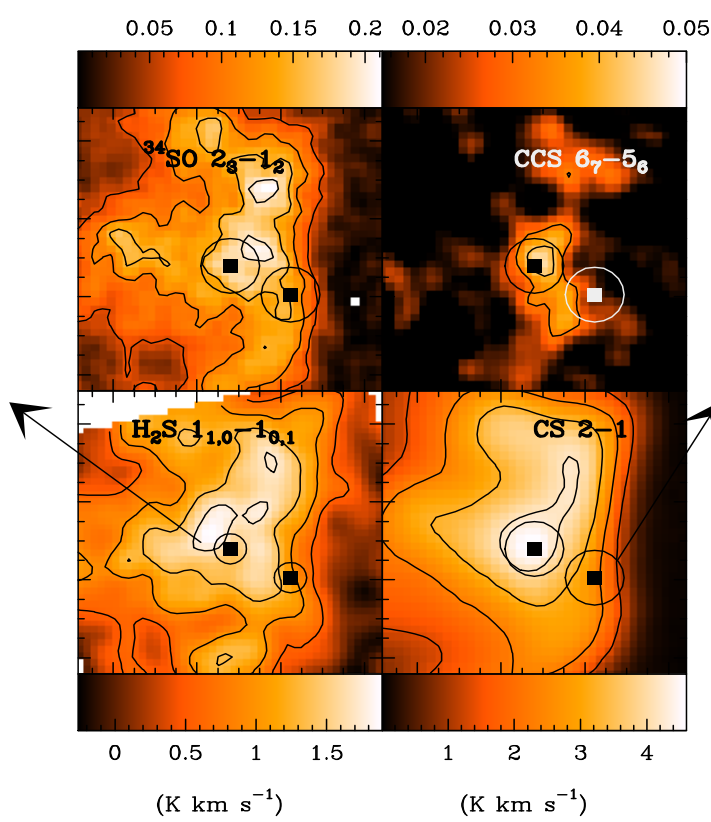

$\mathrm{T}_{\mathrm{mb}}(\mathrm{K})$

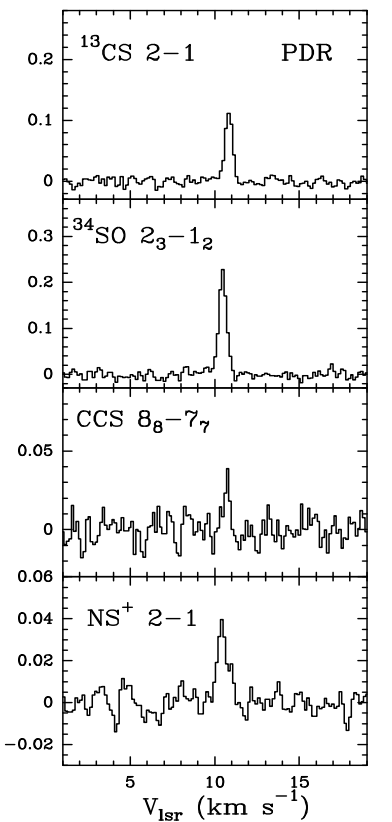

Fig. 1. Integrated intensity maps of the ${ }^{13} \mathrm{CS} 2 \rightarrow 1,{ }^{34} \mathrm{SO} 2_{3} \rightarrow 1_{2}$, CCS $8_{7} \rightarrow 7_{6}$, and $\mathrm{H}_{2} \mathrm{~S} 1_{1,0} \rightarrow 1_{0,1}$ maps as observed with the $30 \mathrm{~m}$ IRAM telescope. The beam of the $30 \mathrm{~m}$ telescope at $3 \mathrm{~mm}\left(H P B W \sim 29^{\prime \prime}\right)$ is indicated by two circles centred on the positions targeted within the Whisper spectral survey. Line contours depict $30-90$ by $15 \%$ levels with respect to the emission peak.

into main-beam temperatures $\left(T_{\mathrm{mb}}\right)$ using the forward and mainbeam efficiencies of the telescope. The resulting spectra were then baseline-corrected and gridded through convolution with a Gaussian to obtain the maps. Beam sizes are roughly in the range $12^{\prime \prime}-25^{\prime \prime}$, depending on the exact frequency of each transition, more precisely, $H P B W\left({ }^{\prime \prime}\right)=2460 / v(\mathrm{GHz})^{2}$.

\section{Molecular inventory}

Taking the high sensitivity WHISPER survey as the observational basis, we have systematically searched for all the S-bearing species included in the Cologne Database for Molecular Spectroscopy (CDMS) catalogue. A complete list of intensities for the detected lines is provided in Table A.1. Figure A.1 depicts the spectra of the detected transitions grouped by species. For the detected species, we have carried out a multi-transitional study that includes lines of different isotopologues, to determine accurate molecular abundances (Tables 1 and 4). In the case of non-detections, an upper limit to the fractional abundance is given. The list of non-detected species is shown in Table 3. A detailed description of the methods used to derive molecular abundances can be found in Sects. 4 and 5 .

The list of S-bearing species detected in the Horsehead includes $\mathrm{CS}, \mathrm{SO}, \mathrm{SO}_{2}$, OCS, $\mathrm{H}_{2} \mathrm{CS}$ (both ortho- and para-), $\mathrm{HDCS}, \mathrm{C}_{2} \mathrm{~S}, \mathrm{HCS}^{+}, \mathrm{SO}^{+}, \mathrm{H}_{2} \mathrm{~S}, \mathrm{~S}_{2} \mathrm{H}, \mathrm{NS}$, and $\mathrm{NS}^{+}$. The detections of $\mathrm{H}_{2} \mathrm{~S}$ and $\mathrm{S}_{2} \mathrm{H}$ were previously reported by Fuente et al. (2017). It is important to note that this list constitutes the most complete inventory of S-bearing species in a PDR thus far. We have not detected $\mathrm{DCS}^{+}$and CCCS towards any of the observed positions, and we provide upper limits for the fractional abundance of these molecules. The detection of $\mathrm{NS}^{+}$ reported here is worth noticing as the first detection of $\mathrm{NS}^{+}$ in a PDR. $\mathrm{NS}^{+}$was recently identified by Cernicharo et al.

\footnotetext{
2 See http://www.iram.es/IRAMES/telescope/telescope Summary/telescope_summary.html
}

(2018) in a variety of dark clouds, with a NS/NS ${ }^{+} \sim 30-50$. However, a NS/NS ${ }^{+}>3000$ was measured in the Orion KL hot core (Cernicharo et al. 2018). We have detected two $\mathrm{NS}^{+}$ lines, $J=2 \rightarrow 1$ at $110198.5 \mathrm{MHz}$ and $J=3 \rightarrow 2$ at $150295.6 \mathrm{MHz}$, with a signal-to-noise ratio $(S / N)>4$ towards the PDR position. Only the line at $110198.5 \mathrm{MHz}$ has been detected towards the core (see Fig. 2).

We present also the first detection of $\mathrm{SO}^{+}$in a low-UV PDR $(\chi<500)$. Detected earlier by Turner $(1992), \mathrm{SO}^{+}$has been detected in variety of regions, which include massive star formation regions, shocked regions, and translucent clouds (Turner 1992, 1994, 1996). Although widely detected, a high abundance of $\mathrm{SO}^{+}$relative to $\mathrm{SO}, \mathrm{X}\left(\mathrm{SO}^{+}\right) / \mathrm{X}(\mathrm{SO})>100$, seems to be related to photon-dominated chemistry and was proposed by Sternberg \& Dalgarno (1995) as a tracer of UV illuminated regions. These high $\mathrm{SO}^{+}$abundances have been previously measured in the PDRs associated to NGC 7023 (Fuente et al. 2003), the Orion Bar (Fuente et al. 2003; Goicoechea et al. 2017), and Monoceros R 2 (Ginard et al. 2012), but this ion has not been detected in a low-UV PDR such as the Horsehead thus far.

\section{Rotational diagrams}

We have used the rotational diagram technique to derive rotational temperatures, $T_{\text {rot }}$, and beam-averaged column densities, $N(\mathrm{X})$. The results are summarized in Table 1 . This method assumes that the population of the molecular energy levels can be characterized by a single temperature, $T_{\text {rot }}$, and that this temperature is uniform along the line of sight. Given the large beam sizes in our observations $\left(\sim 12^{\prime \prime}\right.$ at 200 to $\sim 25^{\prime \prime}$ at $\left.100 \mathrm{GHz}\right)$, the assumption of a single $T_{\text {rot }}$ for each molecule and position is an over-simplification, yet a valid starting point for our analysis. We also assume that the emission from the different species is filling the beam. While this is most likely the case for many species (see Fig. 1), it might bias our results in some cases. 
Table 1. Rotational temperatures, column densities, and abundances derived from rotational diagrams for S-bearing species in the Horsehead nebula.

\begin{tabular}{|c|c|c|c|c|c|c|}
\hline \multirow[b]{2}{*}{ Species } & \multicolumn{3}{|c|}{ PDR } & \multicolumn{3}{|c|}{ Core } \\
\hline & $\begin{array}{l}T_{\text {rot }} \\
(\mathrm{K})\end{array}$ & $\begin{array}{l}N(\mathrm{X}) \\
\left(\mathrm{cm}^{-2}\right)\end{array}$ & $\begin{array}{l}\text { Abundance } \\
\frac{N(\mathrm{X})}{N(\mathrm{H})+2 N\left(\mathrm{H}_{2}\right)}\end{array}$ & $\begin{array}{l}T_{\text {rot }} \\
(\mathrm{K})\end{array}$ & $\begin{array}{c}N(\mathrm{X}) \\
\left(\mathrm{cm}^{-2}\right)\end{array}$ & $\begin{array}{l}\text { Abundance } \\
\frac{N(\mathrm{X})}{N(\mathrm{H})+2 N\left(\mathrm{H}_{2}\right)}\end{array}$ \\
\hline $\mathrm{CS}^{(a)}$ & 6.3 & $(3.0 \pm 0.6) \times 10^{13}$ & $(8.0 \pm 2.0) \times 10^{-10}$ & 5.9 & $(5.3 \pm 1.0) \times 10^{13}$ & $(9.0 \pm 2.0) \times 10^{-10}$ \\
\hline $\mathrm{SO}^{(a)}$ & 11.6 & $(3.2 \pm 0.3) \times 10^{13}$ & $(8.4 \pm 0.8) \times 10^{-10}$ & 8.2 & $(5.0 \pm 0.5) \times 10^{13}$ & $(8.6 \pm 0.9) \times 10^{-10}$ \\
\hline $\mathrm{p}-\mathrm{H}_{2} \mathrm{CS}$ & 4.2 & $(5.0 \pm 3.0) \times 10^{11}$ & $(1.3 \pm 0.8) \times 10^{-11}$ & 8.6 & $(9.0 \pm 1.0) \times 10^{11}$ & $(1.6 \pm 0.2) \times 10^{-11}$ \\
\hline $\mathrm{o}-\mathrm{H}_{2} \mathrm{CS}$ & 8.8 & $(9.0 \pm 3.0) \times 10^{11}$ & $(2.4 \pm 0.8) \times 10^{-11}$ & 8.2 & $(2.5 \pm 0.8) \times 10^{12}$ & $(4.3 \pm 1.4) \times 10^{-11}$ \\
\hline $\mathrm{SO}_{2}$ & 6.5 & $(3.0 \pm 0.6) \times 10^{12}$ & $(8.0 \pm 1.6) \times 10^{-11}$ & 6.9 & $(2.9 \pm 0.6) \times 10^{12}$ & $(5.0 \pm 1.0) \times 10^{-11}$ \\
\hline CCS & 10.3 & $(3.0 \pm 1.0) \times 10^{11}$ & $(8.0 \pm 2.6) \times 10^{-12}$ & 8.1 & $(1.5 \pm 0.7) \times 10^{12}$ & $(2.6 \pm 1.2) \times 10^{-11}$ \\
\hline $\mathrm{HCS}^{+}$ & 11.6 & $(5.0 \pm 0.6) \times 10^{11}$ & $(1.3 \pm 0.2) \times 10^{-11}$ & $10^{(b)}$ & $6 \times 10^{11}$ & $1.0 \times 10^{-11}$ \\
\hline $\mathrm{SO}^{+}$ & 11 & $(6.3 \pm 1.8) \times 10^{11}$ & $(1.7 \pm 0.5) \times 10^{-11}$ & 9 & $(4.5 \pm 0.5) \times 10^{11}$ & $(7.8 \pm 0.9) \times 10^{-12}$ \\
\hline HDCS & $10^{(b)}$ & $(6 \pm 1) \times 10^{11}$ & $(2 \pm 0.4) \times 10^{-11}$ & $10^{(b)}$ & $(1 \pm 0.2) \times 10^{12}$ & $(2 \pm 0.4) \times 10^{-11}$ \\
\hline NS & $10^{(b)}$ & $(4 \pm 0.8) \times 10^{12}$ & $(1 \pm 0.2) \times 10^{-10}$ & $10^{(b)}$ & $(3 \pm 0.6) \times 10^{12}$ & $(5 \pm 1) \times 10^{-11}$ \\
\hline $\mathrm{NS}^{+}$ & $10^{(b)}$ & $(1 \pm 0.2) \times 10^{11}$ & $(3 \pm 0.6) \times 10^{-12}$ & $10^{(b)}$ & $(6 \pm 1) \times 10^{10}$ & $(1 \pm 0.2) \times 10^{-12}$ \\
\hline $\mathrm{DCS}^{+}$ & $10^{(b)}$ & $<1.0 \times 10^{11}$ & $<3 \times 10^{-12}$ & $10^{(b)}$ & $<1.0 \times 10^{11}$ & $<2 \times 10^{-12}$ \\
\hline CCCS & $10^{(b)}$ & $<3.2 \times 10^{11}$ & $<8 \times 10^{-12}$ & $10^{(b)}$ & $<3.2 \times 10^{11}$ & $<5 \times 10^{-12}$ \\
\hline
\end{tabular}

Notes. Abundances are computed assuming $N\left(\mathrm{H}_{2}\right)=1.9 \times 10^{22} \mathrm{~cm}^{-2}$ towards the PDR and $N\left(\mathrm{H}_{2}\right)=2.9 \times 10^{22} \mathrm{~cm}^{-2}$ towards the core from Gerin et al. (2009a). ${ }^{(a)}$ Computed including opacity effects. ${ }^{(b)}$ Since only one transition was detected, we assumed $T_{\text {rot }}=10 \mathrm{~K}$ and fitted the column density to match the observed $T_{\mathrm{MB}}$ assuming LTE.
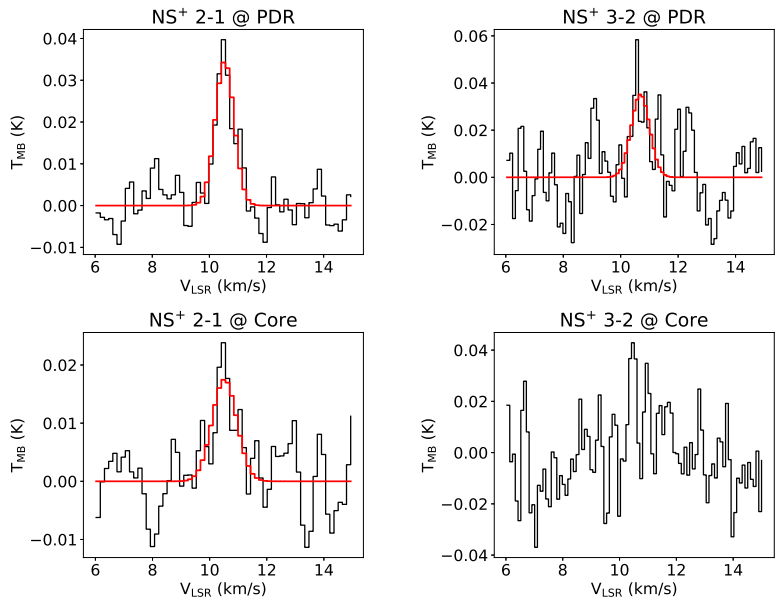

Fig. 2. Spectra of $\mathrm{NS}^{+}$in the PDR and the core. The red line shows the Gaussian fit to the observed line profile.

In its simplest formulation, assuming optically thin emission and the Rayleigh-Jeans limit, the rotation temperature and molecular column density can be derived from the equations

$\ln \left(\frac{N_{\text {up }}}{g_{\text {up }}}\right)=\ln \left(\frac{N_{\text {tot }}}{Q_{\text {rot }}}\right)-\frac{E_{\text {up }}}{k T_{\text {rot }}}$,

$N_{\text {up }}\left(\mathrm{cm}^{-2}\right)=1.94 \times 10^{3} v^{2}(\mathrm{GHz}) W / A_{\mathrm{ul}}\left(\mathrm{s}^{-1}\right)$,

where $W$ is the velocity-integrated line area in units of $\mathrm{K} \mathrm{kms}^{-1}$. We assume that the emission is extended compared with the beam size, that is, there is a beam filling factor of 1 , and consider only $S / N$ (Area) $>5$ detections to compute rotational diagrams.

The assumption of optically thin emission cannot be applied to the abundant species CS and SO. In these cases, we use the expression

$\ln \left(\frac{N_{\text {up }}^{\text {obs }}}{g_{\text {up }}}\right)=\ln \left(\frac{N_{\text {tot }}^{\text {obs }}}{Q_{\text {rot }}}\right)-\frac{E_{\text {up }}}{k T_{\text {rot }}}-\ln \left(C_{\tau}\right)$,
Table 2. Collisional partners and collisional excitation rates for the different species modelled with LVG.

\begin{tabular}{ll}
\hline \hline Species & Collisional excitation rates \\
\hline $\mathrm{CS}$ & Denis-Alpizar et al. (2013) \\
$\mathrm{SO}$ & Lique et al. (2006b) \\
$\mathrm{SO}_{2}$ & Green (1995) \\
$\mathrm{OCS}$ & Green \& Chapman (1978) \\
$\mathrm{H}_{2} \mathrm{CS}$ & Wiesenfeld \& Faure (2013) \\
$\mathrm{HCS}^{+}$ & Flower (1999)
\end{tabular}

Notes. ${ }^{(a)}$ Adapted from $\mathrm{HCO}^{+}$collisional rates.

with

$C_{\tau}=\frac{\tau}{1-e^{-\tau}}$,

being the optical depth correction factor (Goldsmith \& Langer 1999). Line opacities, $\tau$, are derived for each individual line assuming ${ }^{12} \mathrm{C} /{ }^{13} \mathrm{C}=60$ and ${ }^{32} \mathrm{~S} /{ }^{34} \mathrm{~S}=22.5$ (Wilson \& Rood 1994). For a few species, we detected only one transition and we need to assume a certain $T_{\text {rot }}$ to estimate column densities. Based on the results of the rotational diagrams of molecules with similar dipole moments (Table 1 and Fuente et al. 2017; Goicoechea et al. 2006), we assume $T_{\text {rot }}=10 \mathrm{~K}$ in these cases. The same rotational temperature is used to calculate the $3 \sigma$ column density upper limits shown in Table 3.

\section{RADEX models}

We used the molecular excitation code RADEX (van der Tak et al. 2007) to get a more detailed description of the physical conditions and molecular abundances at the two positions observed. RADEX is a non-Local Thermodynamic Equilibrium (LTE) molecular excitation and local radiative transfer code 
Table 3. Upper limits to the column density and abundance of undetected species.

\begin{tabular}{|c|c|c|c|c|c|c|c|}
\hline \multirow[t]{2}{*}{ Molecule } & \multirow{2}{*}{$\begin{array}{c}\text { Freq } \\
(\mathrm{GHz})\end{array}$} & \multirow{2}{*}{$\begin{array}{l}\mathrm{rms}^{(1)} \\
(\mathrm{mK})\end{array}$} & \multirow{2}{*}{$\begin{array}{c}N_{X}^{(2)} \\
\left(\mathrm{cm}^{-2}\right) \\
\end{array}$} & \multicolumn{3}{|c|}{ Abundances wrt $\mathrm{H}_{2}$} & \multirow[t]{2}{*}{ Ref. } \\
\hline & & & & Horsehead & Dark clouds & Hot cores & \\
\hline $\mathrm{S}_{2} \mathrm{H}_{2}$ & 139.885 & 9 & $<8.5 \times 10^{11}$ & $<4.5 \times 10^{-11}$ & & & \\
\hline $\mathrm{HSO}$ & 158.391 & 30 & $<1.5 \times 10^{12}$ & $<7.9 \times 10^{-11}$ & & & \\
\hline $\mathrm{HCS}$ & 161.128 & 30 & $<3.0 \times 10^{13}$ & $<1.6 \times 10^{-9}$ & $\sim 1-5 \times 10^{-10}$ & & Agúndez et al. (2018) \\
\hline HSC & 81.200 & 17 & $<7.5 \times 10^{11}$ & $<3.9 \times 10^{-11}$ & $\sim 6 \times 10^{-12}$ & & Agúndez et al. (2018) \\
\hline HCCS & 88.023 & 4 & $<3.6 \times 10^{11}$ & $<1.9 \times 10^{-11}$ & & & \\
\hline NCS & 91.501 & 4 & $<7.0 \times 10^{11}$ & $<3.7 \times 10^{-11}$ & & & \\
\hline HNCS & 82.102 & 8 & $<4.2 \times 10^{11}$ & $<2.2 \times 10^{-11}$ & $\sim 4 \times 10^{-12}$ & $\sim 8 \times 10^{-12}$ & Adande et al. (2010) \\
\hline $\mathrm{HSCN}$ & 92.230 & 5 & $<4.0 \times 10^{11}$ & $<2.1 \times 10^{-11}$ & $\sim 3.5 \times 10^{-12}$ & $\sim 2 \times 10^{-12}$ & Adande et al. (2010) \\
\hline HCNS & 86.118 & 8 & $<8.0 \times 10^{10}$ & $<4.2 \times 10^{-12}$ & & & \\
\hline HSNC & 87.900 & 3 & $<8.0 \times 10^{10}$ & $<4.2 \times 10^{-12}$ & & & \\
\hline $\mathrm{H}_{2} \mathrm{C}_{3} \mathrm{~S}$ & 85.728 & 8 & $<8.0 \times 10^{12}$ & $<4.2 \times 10^{-10}$ & & & \\
\hline $\mathrm{CH}_{3} \mathrm{SH}$ & 99.185 & 7 & $<1.2 \times 10^{13}$ & $<6.3 \times 10^{-10}$ & & $\sim 4 \times 10^{-9}$ & Majumdar et al. (2016) \\
\hline $\mathrm{CS}^{+}$ & 103.933 & 7 & $<1.5 \times 10^{12}$ & $<7.9 \times 10^{-11}$ & & & \\
\hline $\mathrm{HSCO}^{+}$ & 90.183 & 4 & $<3.5 \times 10^{11}$ & $<1.8 \times 10^{-11}$ & & & \\
\hline $\mathrm{HOCS}^{+}$ & 91.624 & 4 & $<3.5 \times 10^{11}$ & $<1.8 \times 10^{-11}$ & & & \\
\hline $\mathrm{HOSO}^{+}$ & 88.452 & 4 & $<4.5 \times 10^{11}$ & $<1.8 \times 10^{-11}$ & & & \\
\hline
\end{tabular}

Notes. ${ }^{(1)}$ The rms has been calculated for a channel width of $\approx 0.33 \mathrm{~km} \mathrm{~s}^{-1}$. The obtained rms is similar in the two surveyed positions. ${ }^{(2)} 3 \sigma$ upper limits assuming LTE, $T_{\text {rot }}=10 \mathrm{~K}$, and a linewidth of $0.6 \mathrm{~km} \mathrm{~s}^{-1}$. Abundances in the Horsehead were derived assuming $N\left(\mathrm{H}_{2}\right)=1.9 \times 10^{22} \mathrm{~cm}^{-2}$.

Table 4. Mean values for kinetic temperatures, gas densities, column densities, and molecular abundances from RADEX models explored with MCMC.

\begin{tabular}{|c|c|c|c|c|c|c|c|c|}
\hline \multirow[b]{2}{*}{ Species } & \multicolumn{4}{|c|}{ PDR } & \multicolumn{4}{|c|}{ Core } \\
\hline & $\begin{array}{l}T_{\mathrm{K}} \\
(\mathrm{K})\end{array}$ & $\begin{array}{c}n_{\mathrm{H}_{2}} \\
\left(\mathrm{~cm}^{-3}\right)\end{array}$ & $\begin{array}{c}N(\mathrm{X}) \\
\left(\mathrm{cm}^{-2}\right)\end{array}$ & $\begin{array}{l}\text { Abundance } \\
\frac{N(\mathrm{X})}{N(\mathrm{H})+2 N\left(\mathrm{H}_{2}\right)}\end{array}$ & $\begin{array}{l}T_{\mathrm{K}} \\
(\mathrm{K})\end{array}$ & $\begin{array}{c}n_{\mathrm{H}_{2}} \\
\left(\mathrm{~cm}^{-3}\right)\end{array}$ & $\begin{array}{c}N(\mathrm{X}) \\
\left(\mathrm{cm}^{-2}\right)\end{array}$ & $\begin{array}{l}\text { Abundance } \\
\frac{N(\mathrm{X})}{N(\mathrm{H})+2 N\left(\mathrm{H}_{2}\right)}\end{array}$ \\
\hline CS & $58 \pm 10$ & $(5.3 \pm 1.7) \times 10^{4}$ & $(1.7 \pm 0.4) \times 10^{13}$ & $(4.5 \pm 1.1) \times 10^{-10}$ & $27 \pm 6$ & $(9.5 \pm 3.2) \times 10^{4}$ & $(3.9 \pm 0.7) \times 10^{13}$ & $(6.7 \pm 1.2) \times 10^{-10}$ \\
\hline SO & $44 \pm 12$ & $(6.9 \pm 2.2) \times 10^{4}$ & $(1.5 \pm 0.2) \times 10^{13}$ & $(3.9 \pm 0.5) \times 10^{-10}$ & $15 \pm 3$ & $(2.1 \pm 0.7) \times 10^{5}$ & $(3.0 \pm 0.4) \times 10^{13}$ & $(5.2 \pm 0.7) \times 10^{-10}$ \\
\hline $\mathrm{SO}_{2}$ & $58 \pm 10$ & $(7.0 \pm 2.3) \times 10^{4}$ & $(4.4 \pm 0.8) \times 10^{12}$ & $(1.2 \pm 0.2) \times 10^{-10}$ & $25 \pm 8$ & $(1.1 \pm 0.4) \times 10^{5}$ & $(4.3 \pm 0.7) \times 10^{12}$ & $(7.4 \pm 1.2) \times 10^{-11}$ \\
\hline OCS & $61 \pm 10$ & $(7.5 \pm 3.7) \times 10^{4}$ & $(1.9 \pm 0.3) \times 10^{12}$ & $(5.0 \pm 0.8) \times 10^{-11}$ & $24 \pm 6$ & $(1.0 \pm 0.5) \times 10^{5}$ & $(3.0 \pm 0.5) \times 10^{12}$ & $(5.2 \pm 0.9) \times 10^{-11}$ \\
\hline $\mathrm{O}-\mathrm{H}_{2} \mathrm{CS}$ & $59 \pm 10$ & $(5.0 \pm 1.7) \times 10^{4}$ & $(9.0 \pm 1.0) \times 10^{11}$ & $(2.4 \pm 0.3) \times 10^{-11}$ & $18 \pm 6$ & $(9.1 \pm 3.4) \times 10^{4}$ & $(2.4 \pm 0.3) \times 10^{12}$ & $(4.1 \pm 0.5) \times 10^{-11}$ \\
\hline $\mathrm{p}-\mathrm{H}_{2} \mathrm{CS}$ & - & - & - & - & $22 \pm 6$ & $(9.0 \pm 3.4) \times 10^{4}$ & $(9.0 \pm 2.0) \times 10^{11}$ & $(1.6 \pm 0.4) \times 10^{-11}$ \\
\hline $\mathrm{HCS}^{+}$ & $54 \pm 10$ & $(3.0 \pm 0.8) \times 10^{4}$ & $(4.1 \pm 0.8) \times 10^{11}$ & $(1.1 \pm 0.2) \times 10^{-11}$ & - & - & - & - \\
\hline
\end{tabular}

Notes. Abundances are computed assuming $N\left(\mathrm{H}_{2}\right)=1.9 \times 10^{22} \mathrm{~cm}^{-2}$ in the PDR and $N\left(\mathrm{H}_{2}\right)=2.9 \times 10^{22} \mathrm{~cm}^{-2}$ in the core (Gerin et al. 2009a).

that provides line intensities of molecular species for a given combination of $T_{\mathrm{k}}, n_{\mathrm{H}_{2}}$, and $N(\mathrm{X})$. We used a Monte Carlo Markov chain (MCMC) methodology to explore the parameter space using a Bayesian inference approach. Specifically, we used the emcee (Foreman-Mackey et al. 2013) implementation of the Affine Invariant MCMC Ensemble sampler methods by Goodman \& Weare (2010).

When several isotopologues of the same species were detected, as is the case for CS and SO, all the isotopologues were modelled simultaneously assuming the isotopic ratios ${ }^{13} \mathrm{C} /{ }^{12} \mathrm{C}=$ 60 and ${ }^{34} \mathrm{~S} /{ }^{32} \mathrm{~S}=22.5$. Based on our previous knowledge of the region and previous studies by the WHISPER team (Gratier et al. 2013), we decided to assume a Gaussian prior for the kinetic temperature, with mean value and standard deviation $T_{\mathrm{k}}=60 \pm 10 \mathrm{~K}$ in the PDR and $T_{\mathrm{k}}=25 \pm 10 \mathrm{~K}$ in the core, and a log-normal prior for the gas density with mean value and standard deviation $\log _{10}\left(n_{\mathrm{H}_{2}}\left[\mathrm{~cm}^{-3}\right]\right)=4.8 \pm 0.2$ in the PDR and $\log _{10}\left(n_{\mathrm{H}_{2}}\left[\mathrm{~cm}^{-3}\right]\right)=5.0 \pm 0.2$ in the core. We assumed a flat prior for column densities between $10^{9}$ and $10^{15} \mathrm{~cm}^{-2}$. An overview of the collisional partners and collisional excitation rates used for the different species modelled is shown in Table 2. For SO transitions, collisional rates are available either in the range 10-50 K (Lique et al. 2006b) or 60-300 K (Lique et al. 2006a). For our simulations, we used the low temperature collisional rates from Lique et al. (2006b).

Histograms and density plots showing the results of the MCMC analysis are depicted in Fig. B.1. The kinetic temperatures and column densities thus derived are summarized in Table 4. Kinetic temperatures derived from RADEX are systematically higher than those derived from rotational diagrams, which is consistent with sub-thermal excitation. For most molecules, column densities derived from rotational diagrams and RADEX give compatible results, within uncertainties. However, some prominent exceptions are observed. In the PDR, beam-averaged column densities of CS and SO derived from rotational diagrams are two times larger than values derived using RADEX. In the core, the SO abundance derived from rotational diagrams is two times larger than the abundance obtained from RADEX. Since RADEX models take into account nonLTE effects, we consider results from RADEX models to be 


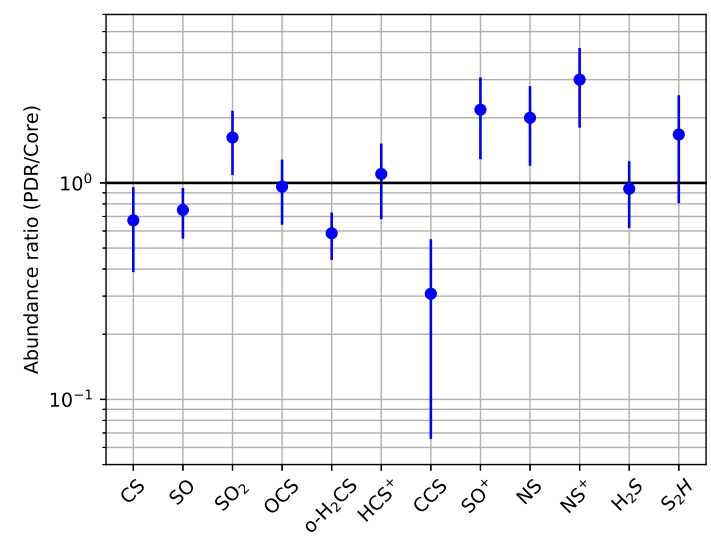

Fig. 3. Ratio between the abundance in the PDR/core position for each species.

better constrained than those coming from rotational diagrams, and we adopt them in the following discussion. We note that, because of the effects of high optical depth, column densities derived using LTE should be lower than those obtained using RADEX. However, we have corrected LTE values for opacity effects (see Eq. (3)). The opacity has been derived from the ratio of the integrated intensity of the main isotopologue over the less abundant one, assuming that $T_{\mathrm{ex}}$ is the same for all the isotopologues. An overestimation of the opacity would lead to an error in the column density calculation. In addition, the assumption of the same rotation temperature for all transitions used in LTE is not always accurate. In any case, the differences between LTE and RADEX models are within the uncertainties inherent to this kind of calculation.

\section{Molecular abundances}

To derive the abundances shown in Tables 1 and 4 we have assumed $N\left(\mathrm{H}_{2}\right)=1.9 \times 10^{22} \mathrm{~cm}^{-2}$ at PDR and $N\left(\mathrm{H}_{2}\right)=$ $2.9 \times 10^{22} \mathrm{~cm}^{-2}$ at the core (Gerin et al. 2009a), and $N(\mathrm{H})=0$ at both positions, meaning that gas is mostly molecular. Given the large beam sizes implied, we consider that this is a valid assumption for the PDR. The $N\left(\mathrm{H}_{2}\right)$ values were derived using a beam of $12^{\prime \prime}$. Following our assumption that the emission is extended and uniform, they should be the same with a larger beam of $25^{\prime \prime}$. We assume this hypothesis to be coherent with our previous studies (Gerin et al. 2009b; Pety et al. 2012; Guzmán et al. 2014).

Our first result is that the fractional abundances and molecular abundance ratios estimated towards the two surveyed positions agree within a factor of approximately three. This gentle gradient is likely due to the limited angular resolution of our observations, which do not resolve the thin outer layers of the PDR. In fact, using interferometric observations, Goicoechea et al. (2006) derived CS abundances towards the outer PDR layers that are approximately five times larger than those presented in this study. Moreover, the line of sight towards the core also intersects the PDR layer in the cloud surface, which makes the detection of chemical gradients more difficult. A factor of two to three, however, can be significant given the high $\mathrm{S} / \mathrm{N}$ in our spectra and the peculiar observational strategy of WHISPER, which avoids calibration and pointing errors. The Cbearing species $\mathrm{C}_{2} \mathrm{~S}$ and $\mathrm{o}-\mathrm{H}_{2} \mathrm{CS}$ present fractional abundances a factor of greater than two higher in the core than towards the PDR (see Fig. 3). In contrast, the O-bearing molecules $\mathrm{SO}, \mathrm{SO}_{2}$, and OCS present similar abundances towards both positions. The same is true for the $\mathrm{HCS}^{+}$. Other species, $\mathrm{S}_{2} \mathrm{H}, \mathrm{SO}^{+}, \mathrm{NS}$, and the recently discovered ion $\mathrm{NS}^{+}$, are more abundant towards the
PDR than towards the core. This work identifies $\mathrm{NS}_{\text {and }} \mathrm{NS}^{+}$as new PDR-like species.

We have unsuccessfully searched for the following sulphuretted compounds: $\mathrm{CS}^{+}, \mathrm{HCS}, \mathrm{HSC}, \mathrm{HCCS}, \mathrm{NCS}, \mathrm{HNCS}$, HSCN, $\mathrm{HCNS}, \mathrm{HSNC}, \mathrm{HSOC}^{+}, \mathrm{HOCS}^{+}, \mathrm{H}_{2} \mathrm{C}_{3} \mathrm{~S}$, and $\mathrm{CH}_{3} \mathrm{SH}$. Some of them, HCS, HSC, HNCS, HSCN, and $\mathrm{CH}_{3} \mathrm{SH}$, were previously detected in the interstellar medium. The radicals HCS and HSC were recently detected towards the dark cloud L483, with abundances of a few $10^{-10}$ and various $10^{-12}$ respectively (Agúndez et al. 2018). HNCS and HSCN were detected in the envelope of Sgr B2 and towards TMC 1 (Adande et al. 2010). The organic sulphur compound $\mathrm{CH}_{3} \mathrm{SH}$ has been detected in hot cores (Sgr B2: Linke et al. 1979, G327.3-0.6: Gibb et al. 2000; Orion: Kolesniková et al. 2014). Majumdar et al. (2016) determined a $\mathrm{CH}_{3} \mathrm{SH}$ abundance of a few $10^{-9}$ towards the hot corino IRAS 16293-2422. The upper limits obtained towards the Horsehead are not good enough to tightly constrain the abundance of these rare species.

We have detected the deuterated sulphur compound HDCS towards the PDR and core positions. This compound was detected by Marcelino et al. (2005) towards the dark cores Barnard $1 \mathrm{~b}$ and TMC1, with $\mathrm{HDCS} / \mathrm{H}_{2} \mathrm{CS} \sim 0.3$, fully consistent with the values derived towards the Horsehead. However, we have not detected $\mathrm{DCS}^{+}$with an upper limit to the $\mathrm{DCS}^{+} / \mathrm{HCS}^{+}$ratio of $\mathrm{DCS}^{+} / \mathrm{HCS}^{+}<0.2$. Fuente et al. (2016) detected $\mathrm{DCS}^{+}$for the first time towards the dark core Barnard $1 \mathrm{~b}$ with $\mathrm{DCS}^{+} / \mathrm{HCS}^{+} \sim 0.2$. The non-detection of $\mathrm{DCS}^{+}$in the core position is roughly consistent with the $\mathrm{DCS}^{+} / \mathrm{HCS}^{+}$ratio measured in Barnard 1b.

\section{Discussion}

We present the abundances of 13 S-bearing species (CS, SO, $\mathrm{SO}_{2}, \mathrm{OCS}, \mathrm{H}_{2} \mathrm{CS}-$ both ortho and para - HDCS, $\mathrm{C}_{2} \mathrm{~S}, \mathrm{HCS}^{+}$, $\mathrm{SO}^{+}, \mathrm{H}_{2} \mathrm{~S}, \mathrm{~S}_{2} \mathrm{H}, \mathrm{NS}$, and $\mathrm{NS}^{+}$) towards the PDR and core positions in the Horsehead nebula. In particular, we highlight the first detection of $\mathrm{NS}^{+}$in a PDR environment, and of $\mathrm{SO}^{+}$in the Horsehead. In the following we discuss the chemistry of these ions and compare the abundances measured towards the Horsehead with those found in other prototypical regions.

\section{1. $\mathrm{NS}^{+}$and $\mathrm{SO}^{+}$}

The chemistry of $\mathrm{NS}$ and $\mathrm{NS}^{+}$is still poorly understood. Cernicharo et al. (2018) explored the main formation mechanisms for these species and their relative importance under typical physical conditions in dark clouds. We show in Fig. 4 the formation channels relevant for a PDR environment like that of the Horsehead nebula, where the $\mathrm{S}^{+}+\mathrm{NH}$ reaction as well as the charge exchange reactions of NS with $\mathrm{C}^{+}$and $\mathrm{S}^{+}$become formation processes of $\mathrm{NS}^{+}$, as $\mathrm{C}^{+}$and $\mathrm{S}^{+}$are the major carbon and sulfur components. Alternatively, the destruction channel of NS by atomic carbon stressed in Cernicharo et al. (2018) becomes less critical than in dense cloud conditions.

The $\mathrm{NS}^{+}$ion is thought to be formed through the reactions

$$
\begin{aligned}
& \mathrm{N}+\mathrm{SH}^{+} \rightarrow \mathrm{NS}^{+}+\mathrm{H}, \\
& \mathrm{N}+\mathrm{SO}^{+} \rightarrow \mathrm{NS}^{+}+\mathrm{O}, \\
& \mathrm{S}^{+}+\mathrm{NH} \rightarrow \mathrm{NS}^{+}+\mathrm{H}, \\
& \mathrm{H}_{2} \mathrm{~S}^{+}+\mathrm{N} \rightarrow \mathrm{NS}^{+}+\mathrm{H}_{2} .
\end{aligned}
$$

These reactions are favoured in UV-illuminated regions. In fact, the ions $\mathrm{S}^{+}, \mathrm{SH}^{+}$, and $\mathrm{SO}^{+}$are thought to be major gasphase sulphur reservoirs in the external layers of the dense, 


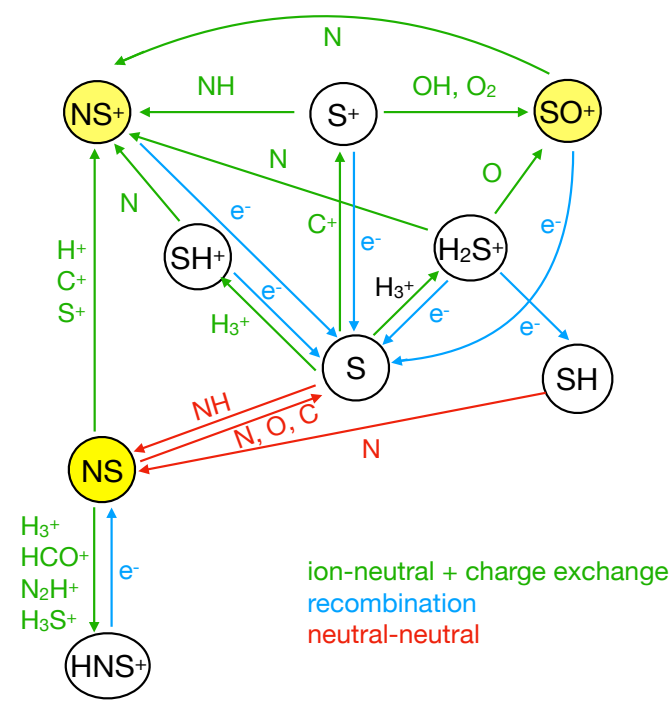

Fig. 4. Gas phase formation and destruction routes of $\mathrm{NS}^{+}$.

high-UV PDRs formed in massive star-forming regions (see e.g. Sternberg \& Dalgarno 1995). The enhanced abundance of $\mathrm{SO}^{+}$in PDRs has been observationally confirmed by single-dish observations towards prototypical PDRs such as NGC 7023, the Orion Bar, and Mon R2 (Fuente et al. 2003; Ginard et al. 2012). The ion $\mathrm{SO}^{+}$is primarily formed through the reaction

$\mathrm{S}^{+}+\mathrm{OH} \rightarrow \mathrm{SO}^{+}+\mathrm{H}$

and is mainly destroyed by dissociative recombination. The radical $\mathrm{OH}$ is the product of the endothermic reaction

$\mathrm{O}+\mathrm{H}_{2} \rightarrow \mathrm{OH}+\mathrm{H}(\Delta E / k \sim 3000 \mathrm{~K})$

and its abundance, and consequently that of $\mathrm{SO}^{+}$, is very sensitive to the gas kinetic temperatures. We have detected for the first time $\mathrm{SO}^{+}$towards the Horsehead, with $N\left(\mathrm{SO}^{+}\right) / N(\mathrm{SO}) \sim$ 0.01-0.04, where gas kinetic temperatures achieves values of $\sim 100-300 \mathrm{~K}$ in the most external layers (Habart et al. 2005). This value is only a few times lower than that measured towards the high-UV and denser PDRs Mon R2 and the Orion Bar (Fuente et al. 2003; Ginard et al. 2012) where the gas kinetic energy might be as high as $\sim 1000 \mathrm{~K}$. The ion $\mathrm{SO}^{+}$has been detected with a lower $N\left(\mathrm{SO}^{+}\right) / N(\mathrm{SO})$ ratio towards the core position, consistent with the presence of a UV-illuminated surface intersecting our line of sight.

The ion $\mathrm{SH}^{+}$is only expected to be detectable in warm regions where the endothermic reaction

$\mathrm{S}^{+}+\mathrm{H}_{2} \rightarrow \mathrm{SH}^{+}+\mathrm{H}(\Delta E / k \sim 9860 \mathrm{~K})$,

can proceed (Zanchet et al. 2019). Interestingly, this reaction becomes exothermic when $\mathrm{H}_{2}$ molecules are in the $v=2$ or higher vibrational levels (Zanchet et al. 2013). The detection of the $\mathrm{SH}^{+}$ emission lines in the high-mass star-forming region W3 IRS5 (Benz et al. 2010) or the Orion Bar (Nagy et al. 2013; Müller et al. 2014) is interpreted in terms of the reactions of $\mathrm{S}^{+}$with vibrationally excited $\mathrm{H}_{2}$ molecules (Zanchet et al. 2019). High angular resolution Atacama Large Millimeter Array (ALMA) images of $\mathrm{SH}^{+}$and $\mathrm{SO}^{+}$in the Orion Bar confirm this interpretation (Goicoechea et al. 2017). In these images, the $\mathrm{SH}^{+}$emission comes only from a thin layer, which is spatially coincident with the $\mathrm{H}_{2} v=1-0 \mathrm{~S}(1)$ emission layer, while $\mathrm{SO}^{+}$extends deeper into the cloud. The ion $\mathrm{SH}^{+}$has also been detected in low density environments. In particular, $\mathrm{SH}^{+}$has been detected in absorption towards Sgr B2 (Menten et al. 2011) and in diffuse clouds was observed toward several Galactic sight lines by Godard et al. (2012), who showed that turbulent dissipation and shocks provide the required energy to form $\mathrm{SH}^{+}$in these very low density clouds. To our knowledge, $\mathrm{SH}^{+}$has not been detected in the Horsehead. This is not surprising since this ion is hardly formed in this nebula. In PDRs, $\mathrm{H}_{2}(v=2)$ and higher vibrational levels are mostly populated by FUV pumping. Since the FUV flux in the Horsehead is not strong, the layer where $\mathrm{H}_{2}(v=2)$ is excited is most likely very narrow, thus strongly diluted to be detected with single-dish telescopes.

Other routes to the formation of $\mathrm{NS}^{+}$involve the ionic species $\mathrm{H}_{2} \mathrm{~S}^{+}$and $\mathrm{S}^{+}$. Because of its low ionization potential, sulfur is expected to be ionized up to $A_{\mathrm{v}} \sim 7 \mathrm{mag}$ (see e.g. Le Gal et al. 2017) and is a plausible precursor of $\mathrm{NS}^{+}$. The abundance of the ion $\mathrm{H}_{2} \mathrm{~S}^{+}$, formed by the reaction $\mathrm{S}+\mathrm{H}_{3}^{+}$, might be important in deeper layers of the molecular cloud depending on the cosmic ray ionization rate (Sternberg \& Dalgarno 1995).

Within this scenario, $\mathrm{NS}^{+}$could be considered as a PDR tracer. This ion has been widely detected in the interstellar medium (Cernicharo et al. 2018). One possibility is that $\mathrm{NS}^{+}$ emission is coming from the UV-illuminated external layers of the cloud. To check this hypothesis, in Fig. 5 we represent the $\mathrm{NS}^{+}$abundance as a function of the total $\mathrm{H}_{2}$ column density for all the reported $\mathrm{NS}^{+}$detections thus far. The $\mathrm{NS}^{+}$abundance is anti-correlated with $N\left(\mathrm{H}_{2}\right)$, as expected if the $\mathrm{NS}^{+}$emission is coming mainly from the cloud surface.

The chemistry of NS is poorly understood as well. This compound is detected in very different environments such as dark clouds and hot cores (Fuente et al. 2016; Cernicharo et al. 2018) as well as towards the Orion Bar (Leurini et al. 2006). Moreover, NS has been detected in comets with an abundance of $0.006-$ $0.12 \%$ relative to water (Calmonte et al. 2016). Ice evaporation might be the origin of NS in hot cores (Vidal et al. 2017; Viti et al. 2004). Other desorption mechanisms (photodesorption, cosmic-rays, grain sputtering) might release some NS molecules to the gas phase even in cold environments. We recall that Fuente et al. (2017) also proposed that these desorption processes would release $\mathrm{H}_{2} \mathrm{~S}$ and $\mathrm{S}_{2} \mathrm{H}$ molecules from the grain surfaces, boosting their abundances in gas phase.

\subsection{Sulphur budget in the Horsehead}

The main reservoir of sulphur in gas phase is still debated. In dark cores, only about $0.05 \%$ is locked in the S-bearing molecules detected at millimeter wavelengths (see the fractional abundances derived for S-bearing species towards the dark cloud TMC1-CP in Table 5). Unfortunately, most of the plausible major sulphur reservoirs such as $\mathrm{S}, \mathrm{HS}$, and $\mathrm{S}_{2}$ are either not observable or very difficult to observe at millimeter wavelengths and one needs to trust to chemical models to estimate the exact amount of sulphur in the gas phase. It is widely accepted that most of the sulphur is locked in icy grain mantles. This is corroborated by the observations of S-bearing species in comets. In a recent compilation, Bockelée-Morvan \& Biver (2017) estimated that the S/O elemental ratio in the comet ice is about $1.5 \%$, which is consistent within a factor of approximately four with the solar value, $\mathrm{H}_{2} \mathrm{~S}$ being the main sulphur reservoir. Hot cores are compact warm regions where the icy mantles evaporate releasing the sulphur budget to the gas. Orion KL is the best-studied hot core, with full spectral surveys at millimeter and submillimeter wavelengths. In Table 5, we show the abundances of sulphur 
Table 5. Comparison of fractional abundances with observational templates.

\begin{tabular}{lcccc}
\hline & HH-PDR & TMC 1-CP & Orion KL & Comets $\left(\%\right.$ wrt $\left.\mathrm{H}_{2} \mathrm{O}\right)$ \\
\hline $\mathrm{CS}$ & $(4.5 \pm 1.1) \times 10^{-10}$ & $1.4 \times 10^{-9}$ & $7.0 \times 10^{-8}$ & $0.02-0.2$ \\
$\mathrm{SO}$ & $(3.9 \pm 0.5) \times 10^{-10}$ & $7.5 \times 10^{-10}$ & $8.0 \times 10^{-8}$ & $0.04-0.3$ \\
$\mathrm{o}-\mathrm{H}_{2} \mathrm{CS}$ & $(2.4 \pm 0.3) \times 10^{-11}$ & $3.0 \times 10^{-10}$ & $5.6 \times 10^{-9}$ & $0.009-0.09$ \\
$\mathrm{OCS}$ & $(5.0 \pm 0.8) \times 10^{-11}$ & $1.1 \times 10^{-9}$ & $5.0 \times 10^{-8}$ & $0.03-0.4$ \\
$\mathrm{CCS}$ & $(8.0 \pm 2.6) \times 10^{-12}$ & $3.5 \times 10^{-9}$ & $8.0 \times 10^{-11}$ & 0.2 \\
$\mathrm{SO}_{2}$ & $(1.2 \pm 0.2) \times 10^{-10}$ & $1.5 \times 10^{-10}$ & $1.9 \times 10^{-7}$ & \\
$\mathrm{SO}^{+}$ & $(1.7 \pm 0.5) \times 10^{-11}$ & & & $0.006-0.012$ \\
$\mathrm{HCS}^{+}$ & $(1.1 \pm 0.2) \times 10^{-11}$ & $1.5 \times 10^{-10}$ & & $0.13-1.5$ \\
$\mathrm{NS}^{+10}$ & $(1.0 \pm 0.2) \times 10^{-10}$ & $4.0 \times 10^{-10}$ & $3.4 \times 10^{-9}$ & \\
$\mathrm{NS}^{+}$ & $(3.0 \pm 0.6) \times 10^{-12}$ & $2.4 \times 10^{-12}$ & & \\
$\mathrm{H}_{2} \mathrm{~S}$ & $(3.1 \pm 0.5) \times 10^{-10}$ & $<2.5 \times 10^{-10}$ & $1.5 \times 10^{-6}$ & \\
$\mathrm{~S}_{2} \mathrm{H}$ & $(8.7 \pm 3.1) \times 10^{-11}$ & & & $4 \times 10^{-6}\left(n\left(\mathrm{H}_{2} \mathrm{O}\right) / n_{\mathrm{H}}=2.7 \times 10^{-4}\right)$ \\
\hline Sulphur budget & $\sim 1.5 \times 10^{-9}$ & $\sim 8 \times 10^{-9}$ & $1.9 \times 10^{-6}$ & \multirow{2}{*}{. } \\
\hline
\end{tabular}

References. TMC1: Agúndez \& Wakelam (2013); Cernicharo et al. (2018) and references therein. Orion-KL: Tercero et al. (2010); Esplugues et al. (2014); Crockett et al. (2014). Comets: Calmonte et al. (2016); Bockelée-Morvan \& Biver (2017).

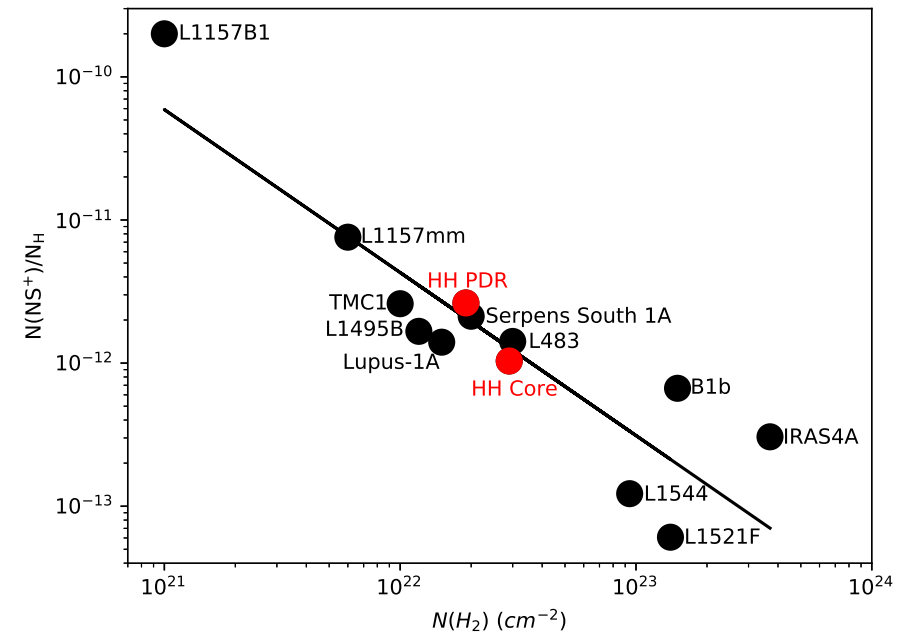

Fig. 5. Abundances of $\mathrm{NS}^{+}$as a function of $N\left(\mathrm{H}_{2}\right)$. Black points corresponds to the detections reported by Cernicharo et al. (2018). Red points corresponds to the core and PDR positions in the Horsehead. The black solid line depicts a linear fit to the data.

species that have been derived in this prototypical hot core. Gas phase $\mathrm{H}_{2} \mathrm{~S}$ is the main sulphur reservoir in this hot core and the elemental gas-phase sulphur abundance is $\sim 1.6 \times 10^{-6}$ (Crockett et al. 2014). Fuente et al. (2019) obtained a similar value for the sulphur gas phase abundance of $\sim 10^{-6}$ in the edges of the TMC1 molecular cloud.

Based on the WHISPER survey, we have determined a significant inventory of sulphur species and their abundances in the Horsehead PDR. Only one $\mathrm{H}_{2} \mathrm{~S}$ line has been observed and the estimated value of the $\mathrm{H}_{2} \mathrm{~S}$ abundance $\left(\sim 0.3-5 \times 10^{-9}\right)$ is hence uncertain. It seems clear, however, that the abundance of gaseous $\mathrm{H}_{2} \mathrm{~S}$ is far from that observed in Orion-KL. One possibility is that a large fraction of $\mathrm{H}_{2} \mathrm{~S}$ remains in the solid state. This is expected since the dust temperature in this PDR is around $\sim 20$ $30 \mathrm{~K}$ in the PDR (Goicoechea et al. 2009), which is below the sublimation temperature of $\mathrm{H}_{2} \mathrm{~S}, \sim 80 \mathrm{~K}$ (Jiménez-Escobar \& Muñoz Caro 2011). The detections of $\mathrm{SO}^{+}$and $\mathrm{NS}^{+}$suggest that a significant fraction of sulphur is in the form of $\mathrm{S}^{+}$in the outer layers of this cloud. Sulphur chemistry is still poorly known, and especially surface chemistry reactions need further understand- ing. Oba et al. (2018) demonstrated that chemical desorption is an efficient mechanism for sulphur-bearing-molecule formation at least in some cases, such as $\mathrm{H}_{2} \mathrm{~S}$. Given the high fraction of sulphur present in a solid state $(\sim 90 \%)$, their work highlights the importance of chemical desorption for understanding the abundances of the different molecular species in the gaseous phase.

Although it has been widely debated what the main sulphur reservoirs in the interstellar medium are, little information is available about the sulphur chemistry in PDRs thus far. Our study shows that $\mathrm{CS}, \mathrm{SO}$, and $\mathrm{H}_{2} \mathrm{~S}$ are the most abundant observable S-bearing molecules in the Horsehead PDR, with similar abundances within one order of magnitude. The total abundance of $\mathrm{S}$ atoms locked in these three species is approximately a few $10^{-9}$, only $\sim 0.1 \%$ of the total number of atoms. Therefore, they do not constitute the main sulphur reservoir. An important fraction of sulphur atoms might be forming icy mantles in these low-UV irradiated regions. Dust grain temperatures in this lowUV PDR are $\sim 20-30 \mathrm{~K}$, lower than the evaporation temperature of $\mathrm{H}_{2} \mathrm{~S}$, the main sulphur reservoir in solid state. In the gas phase, $\mathrm{S}$ and $\mathrm{S}^{+}$are expected to be the main sulphur reservoirs (Le Gal et al. 2017; Goicoechea et al. 2006). Unfortunately the observation of these species is not easy in the (sub-)millimeter wavelength range. Although weak and difficult to interpret, the detection of a sulphur recombination line by Roshi et al. (2014) in NGC 2024 provides evidence for large abundances of $\mathrm{S}$ and $\mathrm{S}^{+}$in the gas phase.

\section{Conclusions}

Photodissociation regions constitute the transition between diffuse clouds, in which sulphur is mainly in the gaseous phase, and cold cores where S-bearing molecules are heavily depleted; therefore they are essential regions to understand sulphur chemistry. Here we present a complete inventory of S-bearing molecules and their abundances in the Horsehead nebula based on the WHISPER survey. Two positions are targeted in this survey, one coincident with a dense core and the other at the PDR-line species peak (PDR). The detections towards the PDR are especially interesting since this is the most complete observational study of S-bearing species in a PDR thus far.

We have detected $13 \mathrm{~S}$-bearing species (CS, SO, $\mathrm{SO}_{2}, \mathrm{OCS}$, $\mathrm{H}_{2} \mathrm{CS}-$ both ortho and para - HDCS, $\mathrm{C}_{2} \mathrm{~S}, \mathrm{HCS}^{+}, \mathrm{SO}^{+}, \mathrm{H}_{2} \mathrm{~S}$, 
$\mathrm{S}_{2} \mathrm{H}$, NS, and $\mathrm{NS}^{+}$) towards the PDR and core positions in the Horsehead nebula.

Two prominent results of this study are the detection of $\mathrm{SO}^{+}$and $\mathrm{NS}^{+}$for the first time in a PDR. Comparing the core and PDR positions, we find a differentiated chemical behaviour between $\mathrm{C}-\mathrm{S}$ and $\mathrm{O}-\mathrm{S}$ bearing species. The $\mathrm{C}-\mathrm{S}$ bearing species $\mathrm{C}_{2} \mathrm{~S}$ and $\mathrm{o}-\mathrm{H}_{2} \mathrm{CS}$ present fractional abundances a factor of greater two higher in the core than in the PDR. In contrast, the $\mathrm{O}-\mathrm{S}$ bearing molecules $\mathrm{SO}, \mathrm{SO}_{2}$, and OCS present similar abundances towards both positions. The same is true for the $\mathrm{HCS}^{+}$. A few molecules are more abundant towards the PDR than towards the core: $\mathrm{S}_{2} \mathrm{H}, \mathrm{SO}^{+}, \mathrm{NS}$, and $\mathrm{NS}^{+}$. Fuente et al. (2017) proposed $\mathrm{S}_{2} \mathrm{H}$ to be a good tracer of low-UV PDRs, where an active grain surface chemistry and gaseous photo-chemistry coexist. We are aware that, with our limited angular resolution, it is doubtful to conclude that $\mathrm{NS}^{+}$is mainly coming from the PDR. Yet $\mathrm{NS}^{+}$ behaves like other $\mathrm{S}$-bearing molecules that have been interpreted as UV tracers, such as $\mathrm{SO}^{+}$and $\mathrm{S}_{2} \mathrm{H}$. Therefore it is worth investigating the link between the enhanced UV-field in PDRs and $\mathrm{NS}^{+}$abundances.

Acknowledgements. We thank the Spanish MINECO for funding support from AYA2016-75066-C2-1/2-P, AYA2017-85111-P and ERC under ERC-2013-SyG, G. A. 610256 NANOCOSMOS. J.R.G. thanks the Spanish MCIU for funding support under grant AYA2017-85111-P. This work was supported by the Programme National "Physique et Chimie du Milieu Interstellaire" (PCMI) of CNRS/INSU with INC/INP co-funded by CEA and CNES.

\section{References}

Adande, G. R., Halfen, D. T., Ziurys, L. M., Quan, D., \& Herbst, E. 2010, ApJ, 725,561

Agúndez, M., \& Wakelam, V. 2013, Chem. Rev., 113, 8710

Agúndez, M., Marcelino, N., Cernicharo, J., \& Tafalla, M. 2018, A\&A, 611, L1

Asplund, M., Grevesse, N., \& Sauval, A. J. 2005, ASP Conf. Ser., 336, 25

Benz, A. O., Bruderer, S., van Dishoeck, E. F., et al. 2010, A\&A, 521, L35

Bockelée-Morvan, D., \& Biver, N. 2017, Phil. Trans. R. Soc. London, Ser. A, 375,20160252

Boogert, A. C. A., Schutte, W. A., Helmich, F. P., Tielens, A. G. G. M., \& Wooden, D. H. 1997, A\&A, 317, 929

Calmonte, U., Altwegg, K., Balsiger, H., et al. 2016, MNRAS, 462, S253

Cernicharo, J., Lefloch, B., Agúndez, M., et al. 2018, ApJ, 853, L22

Crockett, N. R., Bergin, E. A., Neill, J. L., et al. 2014, ApJ, 787, 112

Cuadrado, S., Goicoechea, J. R., Pilleri, P., et al. 2015, A\&A, 575, A82

Cuadrado, S., Goicoechea, J. R., Cernicharo, J., et al. 2017, A\&A, 603, A124

Denis-Alpizar, O., Stoecklin, T., Halvick, P., \& Dubernet, M.-L. 2013, J. Chem. Phys., 139, 204304

Draine, B. T. 1978, ApJS, 36, 595

Esplugues, G. B., Viti, S., Goicoechea, J. R., \& Cernicharo, J. 2014, A\&A, 567, A95

Flower, D. R. 1999, MNRAS, 305, 651

Foreman-Mackey, D., Hogg, D. W., Lang, D., \& Goodman, J. 2013, PASP, 125, 306

Fuente, A., Martin-Pintado, J., Cernicharo, J., \& Bachiller, R. 1993, A\&A, 276, 473

Fuente, A., Martin-Pintado, J., Neri, R., Rogers, C., \& Moriarty-Schieven, G. 1996, A\&A, 310, 286

Fuente, A., Martin-Pintado, J., Rodriguez-Fernández, N. J., Cernicharo, J., \& Gerin, M. 2000, A\&A, 354, 1053

Fuente, A., Cernicharo, J., Roueff, E., et al. 2016, A\&A, 593, A94

Fuente, A., Goicoechea, J. R., Pety, J., et al. 2017, ApJ, 851, L49

Fuente, A., Navarro, D. G., Caselli, P., et al. 2019, A\&A, 624, A105

Fuente, A., Rodríguez-Franco, A., García-Burillo, S., Martín-Pintado, J., \& Black, J. H. 2003, A\&A, 406, 899

Geballe, T. R., Baas, F., Greenberg, J. M., \& Schutte, W. 1985, A\&A, 146, L6 Gerin, M., Goicoechea, J. R., Pety, J., \& Hily-Blant, P. 2009a, A\&A, 494, 977

Gerin, M., Pety, J., \& Goicoechea, J. R. 2009b, ASP Conf. Ser., 417, 165

Gibb, E., Nummelin, A., Irvine, W. M., Whittet, D. C. B., \& Bergman, P. 2000, ApJ, 545, 309
Ginard, D., González-García, M., Fuente, A., et al. 2012, A\&A, 543, A27

Godard, B., Falgarone, E., Gerin, M., et al. 2012, A\&A, 540, A87

Goicoechea, J. R., Pety, J., Gerin, M., et al. 2006, A\&A, 456, 565 Goicoechea, J. R., Compiègne, M., \& Habart, E. 2009, ApJ, 699, L165 Goicoechea, J. R., Pety, J., Cuadrado, S., et al. 2016, Nature, 537, 207 Goicoechea, J. R., Cuadrado, S., Pety, J., et al. 2017, A\&A, 601, L9 Goldsmith, P. F., \& Langer, W. D. 1999, ApJ, 517, 209

Goodman, J., \& Weare, J. 2010, Comm. App. Math. Comp. Sci., 5, 65

Gratier, P., Pety, J., Guzmán, V., et al. 2013, A\&A, 557, A101

Green, S. 1995, ApJS, 100, 213

Green, S., \& Chapman, S. 1978, ApJS, 37, 169

Guzmán, V., Pety, J., Goicoechea, J. R., Gerin, M., \& Roueff, E. 2011, A\&A, 534, A49

Guzmán, V., Pety, J., Gratier, P., et al. 2012, A\&A, 543, L1

Guzmán, V. V., Goicoechea, J. R., Pety, J., et al. 2013, A\&A, 560, A73

Guzmán, V. V., Pety, J., Gratier, P., et al. 2014, Faraday Discuss., 168, 103

Guzmán, V. V., Pety, J., Goicoechea, J. R., et al. 2015, ApJ, 800, L33

Habart, E., Abergel, A., Walmsley, C. M., Teyssier, D., \& Pety, J. 2005, A\&A, 437, 177

Jiménez-Escobar, A., \& Muñoz Caro G. M. 2011, A\&A, 536, A91

Jiménez-Escobar, A., Muñoz Caro, G. M., \& Chen, Y.-J. 2014, MNRAS, 443, 343

Kolesniková, L., Tercero, B., Cernicharo, J., et al. 2014, ApJ, 784, L7

Le Gal, R., Herbst, E., Dufour, G., et al. 2017, A\&A, 605, A88

Leurini, S., Rolffs, R., Thorwirth, S., et al. 2006, A\&A, 454, L47

Linke, R. A., Frerking, M. A., \& Thaddeus, P. 1979, ApJ, 234, L139

Lique, F., Dubernet, M.-L., Spielfiedel, A., \& Feautrier, N. 2006a, A\&A, 450, 399

Lique, F., Spielfiedel, A., \& Cernicharo, J. 2006b, A\&A, 451, 1125

Majumdar, L., Gratier, P., Vidal, T., et al. 2016, MNRAS, 458, 1859

Marcelino, N., Cernicharo, J., Roueff, E., Gerin, M., \& Mauersberger, R. 2005, ApJ, 620, 308

Menten, K. M., Wyrowski, F., Belloche, A., et al. 2011, A\&A, 525, A77

Minissale, M., Dulieu, F., Cazaux, S., \& Hocuk, S. 2016, A\&A, 585, A24

Müller, H. S. P., Goicoechea, J. R., Cernicharo, J., et al. 2014, A\&A, 569, L5

Nagy, Z., Van der Tak, F. F. S., Ossenkopf, V., et al. 2013, A\&A, 550, A96

Neufeld, D. A., Godard, B., Gerin, M., et al. 2015, A\&A, 577, A49

Oba, Y., Tomaru, T., Lamberts, T., Kouchi, A., \& Watanabe, N. 2018, Nat. Astron., 2, 228

Palumbo, M. E., Tielens, A. G. G. M., \& Tokunaga, A. T. 1995, ApJ, 449, 674

Penzias, A. A., \& Burrus, C. A. 1973, ARA\&A, 11, 51

Pety, J., Teyssier, D., Fossé, D., et al. 2005, A\&A, 435, 885

Pety, J., Goicoechea, J. R., Hily-Blant, P., Gerin, M., \& Teyssier, D. 2007, A\&A, 464, L41

Pety, J., Gratier, P., Guzmán, V., et al. 2012, A\&A, 548, A68

Pilleri, P., Fuente, A., Cernicharo, J., et al. 2012, A\&A, 544, A110

Pilleri, P., Treviño-Morales, S., Fuente, A., et al. 2013, A\&A, 554, A87

Pilleri, P., Fuente, A., Gerin, M., et al. 2014, A\&A, 561, A69

Roshi, D. A., Goss, W. M., \& Jeyakumar, S. 2014, ApJ, 793, 83

Sternberg, A., \& Dalgarno, A. 1995, ApJS, 99, 565

Tercero, B., Cernicharo, J., Pardo, J. R., \& Goicoechea, J. R. 2010, A\&A, 517, A96

Tieftrunk, A., Pineau des Forets, G., Schilke, P., \& Walmsley, C. M. 1994, A\&A, 289,579

Treviño-Morales, S. P., Pilleri, P., Fuente, A., et al. 2014, A\&A, 569, A19

Treviño-Morales, S. P., Fuente, A., Sánchez-Monge, Á., et al. 2016, A\&A, 593, L12

Turner, B. E. 1992, ApJ, 396, L107

Turner, B. E. 1994, ApJ, 430, 727

Turner, B. E. 1996, ApJ, 468, 694

van der Tak, F. F. S., Black, J. H., Schöier, F. L., Jansen, D. J., \& van Dishoeck, E. F. 2007, A\&A, 468, 627

Vastel, C., Quénard, D., Le Gal, R., et al. 2018, MNRAS, 478, 5514

Vidal, T. H. G., Loison, J.-C., Jaziri, A. Y., et al. 2017, MNRAS, 469, 435

Viti, S., Collings, M. P., Dever, J. W., McCoustra, M. R. S., \& Williams, D. A. 2004, MNRAS, 354, 1141

Wakelam, V., Caselli, P., Ceccarelli, C., Herbst, E., \& Castets, A. 2004, A\&A, 422, 159

Wiesenfeld, L., \& Faure, A. 2013, MNRAS, 432, 2573

Wilson, T. L., \& Rood, R. 1994, ARA\&A, 32, 191

Zanchet, A., Agúndez, M., Herrero, V. J., Aguado, A., \& Roncero, O. 2013, AJ, 146,125

Zanchet, A., Lique, F., Roncero, O., Goicoechea, J. R., \& Bulut, N. 2019, A\&A, 626, A103 
Appendix A: Additional table and figure

Table A.1. Gaussian fits to the IRAM $30 \mathrm{~m}$ lines.

\begin{tabular}{|c|c|c|c|c|c|c|c|c|c|}
\hline & \multirow[b]{3}{*}{$\begin{array}{l}2 \rightarrow 1 \\
3 \rightarrow 2 \\
5 \rightarrow 4\end{array}$} & \multicolumn{4}{|c|}{ PDR } & \multicolumn{4}{|c|}{ Core } \\
\hline & & \multirow{2}{*}{$\begin{array}{l}T_{\mathrm{MB}} \\
(\mathrm{K})\end{array}$} & \multicolumn{2}{|c|}{$\begin{array}{l}v_{\mathrm{LSR}} \\
\quad\left(\mathrm{km} \mathrm{s}^{-1}\right)\end{array}$} & \multirow{2}{*}{$\begin{array}{c}\int \begin{array}{c}T_{\mathrm{MB}} \mathrm{d} v \\
\left(\mathrm{~K} \mathrm{~km} \mathrm{~s}^{-1}\right)\end{array} \\
2.440 \pm 0.003 \\
1.705 \pm 0.009 \\
0.592 \pm 0.007\end{array}$} & \multirow{2}{*}{$\begin{array}{l}T_{\mathrm{MB}} \\
(\mathrm{K}) \\
5.60 \\
4.70 \\
1.20\end{array}$} & \multicolumn{2}{|c|}{$\begin{array}{l}v_{\mathrm{LSR}} \\
\quad\left(\mathrm{km} \mathrm{s}^{-1}\right)\end{array}$} & \multirow{2}{*}{$\begin{array}{c}\iint_{\left(\mathrm{K} \mathrm{km} \mathrm{s}^{-1}\right)} T_{\mathrm{MB}} \mathrm{d} v \\
4.752 \pm 0.004 \\
3.662 \pm 0.007 \\
0.805 \pm 0.007\end{array}$} \\
\hline $\mathrm{CS}$ & & & $\begin{array}{l}10.70 \pm 0.01 \\
10.67 \pm 0.01 \\
10.65 \pm 0.01\end{array}$ & $\begin{array}{l}0.76 \pm 0.01 \\
0.74 \pm 0.01 \\
0.59 \pm 0.01\end{array}$ & & & $\begin{array}{l}10.67 \pm 0.01 \\
10.63 \pm 0.01 \\
10.61 \pm 0.01\end{array}$ & $\begin{array}{l}0.79 \pm 0.01 \\
0.74 \pm 0.01 \\
0.63 \pm 0.01\end{array}$ & \\
\hline $\mathrm{C}^{34} \mathrm{~S}$ & $\begin{array}{l}2 \rightarrow 1 \\
3 \rightarrow 2 \\
5 \rightarrow 4\end{array}$ & $\begin{array}{l}0.41 \\
0.34 \\
0.05\end{array}$ & $\begin{array}{l}10.72 \pm 0.01 \\
10.72 \pm 0.01 \\
10.57 \pm 0.06\end{array}$ & $\begin{array}{l}0.55 \pm 0.01 \\
0.54 \pm 0.03 \\
0.46 \pm 0.12\end{array}$ & $\begin{array}{l}0.241 \pm 0.003 \\
0.195 \pm 0.008 \\
0.027 \pm 0.006\end{array}$ & $\begin{array}{l}0.78 \\
0.49 \\
-\end{array}$ & $\begin{array}{c}10.70 \pm 0.01 \\
10.67 \pm 0.01 \\
-\end{array}$ & $\begin{array}{c}0.61 \pm 0.01 \\
0.56 \pm 0.01 \\
-\end{array}$ & $\begin{array}{c}0.505 \pm 0.002 \\
0.291 \pm 0.007 \\
-\end{array}$ \\
\hline${ }^{13} \mathrm{CS}$ & $\begin{array}{l}2 \rightarrow 1 \\
3 \rightarrow 2\end{array}$ & $\begin{array}{l}0.11 \\
0.05\end{array}$ & $\begin{array}{l}10.71 \pm 0.01 \\
10.61 \pm 0.07\end{array}$ & $\begin{array}{l}0.52 \pm 0.03 \\
0.66 \pm 0.16\end{array}$ & $\begin{array}{l}0.063 \pm 0.003 \\
0.037 \pm 0.008\end{array}$ & $\begin{array}{l}0.20 \\
0.16\end{array}$ & $\begin{array}{l}10.70 \pm 0.01 \\
10.62 \pm 0.01\end{array}$ & $\begin{array}{l}0.58 \pm 0.02 \\
0.51 \pm 0.04\end{array}$ & $\begin{array}{l}0.127 \pm 0.003 \\
0.088 \pm 0.006\end{array}$ \\
\hline $\mathrm{C}^{33} \mathrm{~S}$ & $\begin{array}{l}2 \rightarrow 1 \\
3 \rightarrow 2 \\
\end{array}$ & $\begin{array}{c}0.07 \\
- \\
\end{array}$ & $\begin{array}{c}11.50 \pm 0.02 \\
-\end{array}$ & $\begin{array}{c}0.48 \pm 0.05 \\
-\end{array}$ & $\begin{array}{c}0.034 \pm 0.003 \\
-\end{array}$ & $\begin{array}{l}0.09 \\
0.08\end{array}$ & $\begin{array}{l}11.46 \pm 0.02 \\
10.92 \pm 0.05\end{array}$ & $\begin{array}{l}0.74 \pm 0.05 \\
0.56 \pm 0.09\end{array}$ & $\begin{array}{l}0.074 \pm 0.004 \\
0.046 \pm 0.007\end{array}$ \\
\hline$\overline{\mathrm{CCS}}$ & $\begin{array}{l}6_{7} \rightarrow 5_{6} \\
7_{6} \rightarrow 6_{5} \\
7_{7} \rightarrow 6_{6} \\
7_{8} \rightarrow 6_{7} \\
8_{7} \rightarrow 7_{6} \\
8_{8} \rightarrow 7_{7}\end{array}$ & $\begin{array}{c}0.09 \\
0.04 \\
0.04 \\
0.07 \\
- \\
0.04\end{array}$ & $\begin{array}{c}10.76 \pm 0.04 \\
10.76 \pm 0.03 \\
10.73 \pm 0.03 \\
10.71 \pm 0.02 \\
- \\
10.74 \pm 0.03\end{array}$ & $\begin{array}{c}0.42 \pm 0.09 \\
0.35 \pm 0.06 \\
0.40 \pm 0.07 \\
0.48 \pm 0.03 \\
- \\
0.32 \pm 0.08\end{array}$ & $\begin{array}{c}0.042 \pm 0.008 \\
0.014 \pm 0.002 \\
0.017 \pm 0.003 \\
0.036 \pm 0.002 \\
- \\
0.013 \pm 0.002\end{array}$ & $\begin{array}{l}0.31 \\
0.09 \\
0.08 \\
0.23 \\
0.08 \\
0.07 \\
\end{array}$ & $\begin{array}{l}10.70 \pm 0.01 \\
10.70 \pm 0.01 \\
10.68 \pm 0.01 \\
10.69 \pm 0.01 \\
10.76 \pm 0.02 \\
10.68 \pm 0.02 \\
\end{array}$ & $\begin{array}{l}0.44 \pm 0.03 \\
0.37 \pm 0.04 \\
0.47 \pm 0.03 \\
0.47 \pm 0.01 \\
0.33 \pm 0.05 \\
0.39 \pm 0.05\end{array}$ & $\begin{array}{c}0.146 \pm 0.007 \\
0.037 \pm 0.003 \\
0.042 \pm 0.002 \\
0.117 \pm 0.002 \\
0.030 \pm 0.003 \\
0.031 \pm 0.003\end{array}$ \\
\hline$\overline{\mathrm{OCS}}$ & $\begin{array}{l}7 \rightarrow 6 \\
8 \rightarrow 7 \\
9 \rightarrow 8 \\
11 \rightarrow 10 \\
12 \rightarrow 11\end{array}$ & $\begin{array}{c}0.02 \\
0.04 \\
0.05 \\
- \\
-\end{array}$ & $\begin{array}{c}10.72 \pm 0.06 \\
10.76 \pm 0.02 \\
10.66 \pm 0.05 \\
- \\
-\end{array}$ & $\begin{array}{c}0.57 \pm 0.11 \\
0.40 \pm 0.04 \\
0.48 \pm 0.14 \\
- \\
-\end{array}$ & $\begin{array}{c}0.014 \pm 0.003 \\
0.019 \pm 0.002 \\
0.024 \pm 0.005 \\
- \\
-\end{array}$ & $\begin{array}{l}0.06 \\
0.05 \\
0.07 \\
0.05 \\
0.08\end{array}$ & $\begin{array}{l}10.69 \pm 0.04 \\
10.62 \pm 0.02 \\
10.76 \pm 0.03 \\
10.60 \pm 0.04 \\
10.50 \pm 0.05\end{array}$ & $\begin{array}{l}0.75 \pm 0.09 \\
0.61 \pm 0.05 \\
0.45 \pm 0.01 \\
0.41 \pm 0.09 \\
0.59 \pm 0.14\end{array}$ & $\begin{array}{c}0.045 \pm 0.005 \\
0.033 \pm 0.002 \\
0.034 \pm 0.004 \\
0.023 \pm 0004 \\
0.048 \pm 0.009\end{array}$ \\
\hline $\mathrm{p}-\mathrm{H}_{2} \mathrm{CS}$ & $\begin{array}{l}3_{03} \rightarrow 2_{02} \\
4_{04} \rightarrow 3_{03} \\
6_{06} \rightarrow 5_{05}\end{array}$ & $\begin{array}{c}0.08 \\
0.05 \\
-\end{array}$ & $\begin{array}{c}10.72 \pm 0.02 \\
10.78 \pm 0.03 \\
-\end{array}$ & $\begin{array}{c}0.52 \pm 0.04 \\
0.30 \pm 0.08 \\
-\end{array}$ & $\begin{array}{c}0.046 \pm 0.003 \\
0.017 \pm 0.004 \\
-\end{array}$ & $\begin{array}{l}0.23 \\
0.23 \\
0.04\end{array}$ & $\begin{array}{l}10.65 \pm 0.01 \\
10.68 \pm 0.02 \\
10.76 \pm 0.05\end{array}$ & $\begin{array}{l}0.55 \pm 0.02 \\
0.46 \pm 0.03 \\
0.70 \pm 0.11\end{array}$ & $\begin{array}{l}0.137 \pm 0.003 \\
0.113 \pm 0.005 \\
0.032 \pm 0.005\end{array}$ \\
\hline $\mathrm{o}-\mathrm{H}_{2} \mathrm{CS}$ & $\begin{array}{l}3_{13} \rightarrow 2_{12} \\
3_{12} \rightarrow 2_{11} \\
4_{14} \rightarrow 3_{13} \\
4_{13} \rightarrow 3_{12}\end{array}$ & $\begin{array}{l}0.13 \\
0.14 \\
0.16 \\
0.10\end{array}$ & $\begin{array}{l}10.68 \pm 0.01 \\
10.75 \pm 0.05 \\
10.65 \pm 0.02 \\
10.84 \pm 0.02\end{array}$ & $\begin{array}{l}0.47 \pm 0.03 \\
0.47 \pm 0.03 \\
0.49 \pm 0.04 \\
0.44 \pm 0.05\end{array}$ & $\begin{array}{l}0.066 \pm 0.003 \\
0.071 \pm 0.003 \\
0.084 \pm 0.005 \\
0.046 \pm 0.005\end{array}$ & $\begin{array}{l}0.33 \\
0.30 \\
0.31 \\
0.28\end{array}$ & $\begin{array}{l}10.65 \pm 0.01 \\
10.71 \pm 0.01 \\
10.61 \pm 0.01 \\
10.80 \pm 0.02\end{array}$ & $\begin{array}{l}0.51 \pm 0.01 \\
0.55 \pm 0.01 \\
0.52 \pm 0.02 \\
0.43 \pm 0.02\end{array}$ & $\begin{array}{l}0.179 \pm 0.003 \\
0.177 \pm 0.003 \\
0.173 \pm 0.005 \\
0.131 \pm 0.005\end{array}$ \\
\hline HDCS & $\begin{array}{l}3_{03} \rightarrow 2_{02} \\
3_{13} \rightarrow 2_{12} \\
3_{12} \rightarrow 2_{11}\end{array}$ & $\begin{array}{c}- \\
- \\
0.05\end{array}$ & $\begin{array}{c}- \\
- \\
10.79 \pm 0.02\end{array}$ & $\begin{array}{c}- \\
- \\
0.31 \pm 0.05\end{array}$ & $\begin{array}{c}- \\
- \\
0.018 \pm 0.002\end{array}$ & $\begin{array}{c}0.06 \\
- \\
0.05\end{array}$ & $\begin{array}{c}10.63 \pm 0.02 \\
- \\
10.79 \pm 0.02\end{array}$ & $\begin{array}{c}0.34 \pm 0.04 \\
- \\
0.50 \pm 0.08\end{array}$ & $\begin{array}{c}0.022 \pm 0.002 \\
- \\
0.027 \pm 0.003\end{array}$ \\
\hline & $\begin{array}{l}2 \rightarrow 1 \\
5 \rightarrow 4 \\
6 \rightarrow 5\end{array}$ & $\begin{array}{l}0.13 \\
0.11 \\
0.06\end{array}$ & $\begin{array}{l}10.75 \pm 0.02 \\
10.75 \pm 0.03 \\
10.64 \pm 0.05\end{array}$ & $\begin{array}{l}0.73 \pm 0.03 \\
0.62 \pm 0.07 \\
0.65 \pm 0.14\end{array}$ & $\begin{array}{l}0.102 \pm 0.004 \\
0.069 \pm 0.007 \\
0.039 \pm 0.006\end{array}$ & $\begin{array}{c}0.11 \\
- \\
-\end{array}$ & $\begin{array}{c}10.75 \pm 0.01 \\
- \\
-\end{array}$ & $\begin{array}{c}0.66 \pm 0.03 \\
- \\
-\end{array}$ & $\begin{array}{c}0.073 \pm 0.003 \\
- \\
-\end{array}$ \\
\hline $\mathrm{SO}$ & $\begin{array}{l}2_{2} \rightarrow 1_{1} \\
2_{3} \rightarrow 1_{2} \\
3_{2} \rightarrow 2_{1} \\
3_{3} \rightarrow 2_{2} \\
3_{4} \rightarrow 2_{3} \\
4_{3} \rightarrow 3_{2} \\
5_{4} \rightarrow 4_{3} \\
5_{5} \rightarrow 4_{4} \\
5_{6} \rightarrow 4_{5}\end{array}$ & $\begin{array}{l}0.45 \\
3.20 \\
0.55 \\
0.70 \\
2.40 \\
0.55 \\
0.34 \\
0.34 \\
1.23 \\
\end{array}$ & $\begin{array}{l}10.72 \pm 0.01 \\
10.73 \pm 0.01 \\
10.73 \pm 0.01 \\
10.72 \pm 0.01 \\
10.68 \pm 0.01 \\
10.69 \pm 0.01 \\
10.69 \pm 0.01 \\
10.70 \pm 0.01 \\
10.68 \pm 0.01\end{array}$ & $\begin{array}{l}0.50 \pm 0.01 \\
0.58 \pm 0.01 \\
0.48 \pm 0.01 \\
0.43 \pm 0.01 \\
0.56 \pm 0.01 \\
0.52 \pm 0.02 \\
0.63 \pm 0.01 \\
0.45 \pm 0.01 \\
0.62 \pm 0.01 \\
\end{array}$ & $\begin{array}{l}0.241 \pm 0.005 \\
2.004 \pm 0.003 \\
0.285 \pm 0.004 \\
0.317 \pm 0.005 \\
1.466 \pm 0.005 \\
0.305 \pm 0.011 \\
0.225 \pm 0.003 \\
0.166 \pm 0.007 \\
0.812 \pm 0.007 \\
\end{array}$ & $\begin{array}{l}0.50 \\
4.50 \\
0.73 \\
1.10 \\
4.40 \\
0.92 \\
0.40 \\
0.40 \\
1.48 \\
\end{array}$ & $\begin{array}{l}10.66 \pm 0.01 \\
10.66 \pm 0.01 \\
10.64 \pm 0.01 \\
10.61 \pm 0.01 \\
10.61 \pm 0.01 \\
10.60 \pm 0.01 \\
10.61 \pm 0.01 \\
10.59 \pm 0.01 \\
10.62 \pm 0.01\end{array}$ & $\begin{array}{l}0.55 \pm 0.08 \\
0.65 \pm 0.01 \\
0.50 \pm 0.01 \\
0.45 \pm 0.01 \\
0.58 \pm 0.01 \\
0.45 \pm 0.02 \\
0.59 \pm 0.02 \\
0.48 \pm 0.02 \\
0.60 \pm 0.01 \\
\end{array}$ & $\begin{array}{l}0.293 \pm 0.003 \\
3.151 \pm 0.003 \\
0.392 \pm 0.005 \\
0.521 \pm 0.005 \\
2.731 \pm 0.007 \\
0.442 \pm 0.013 \\
0.254 \pm 0.007 \\
0.204 \pm 0.007 \\
0.944 \pm 0.006 \\
\end{array}$ \\
\hline
\end{tabular}

Notes. Tabulated errors are the numerical errors of the Gaussian fitting. Other kinds of errors such as calibration or pointing errors are not considered. 
Table A.1. continued.

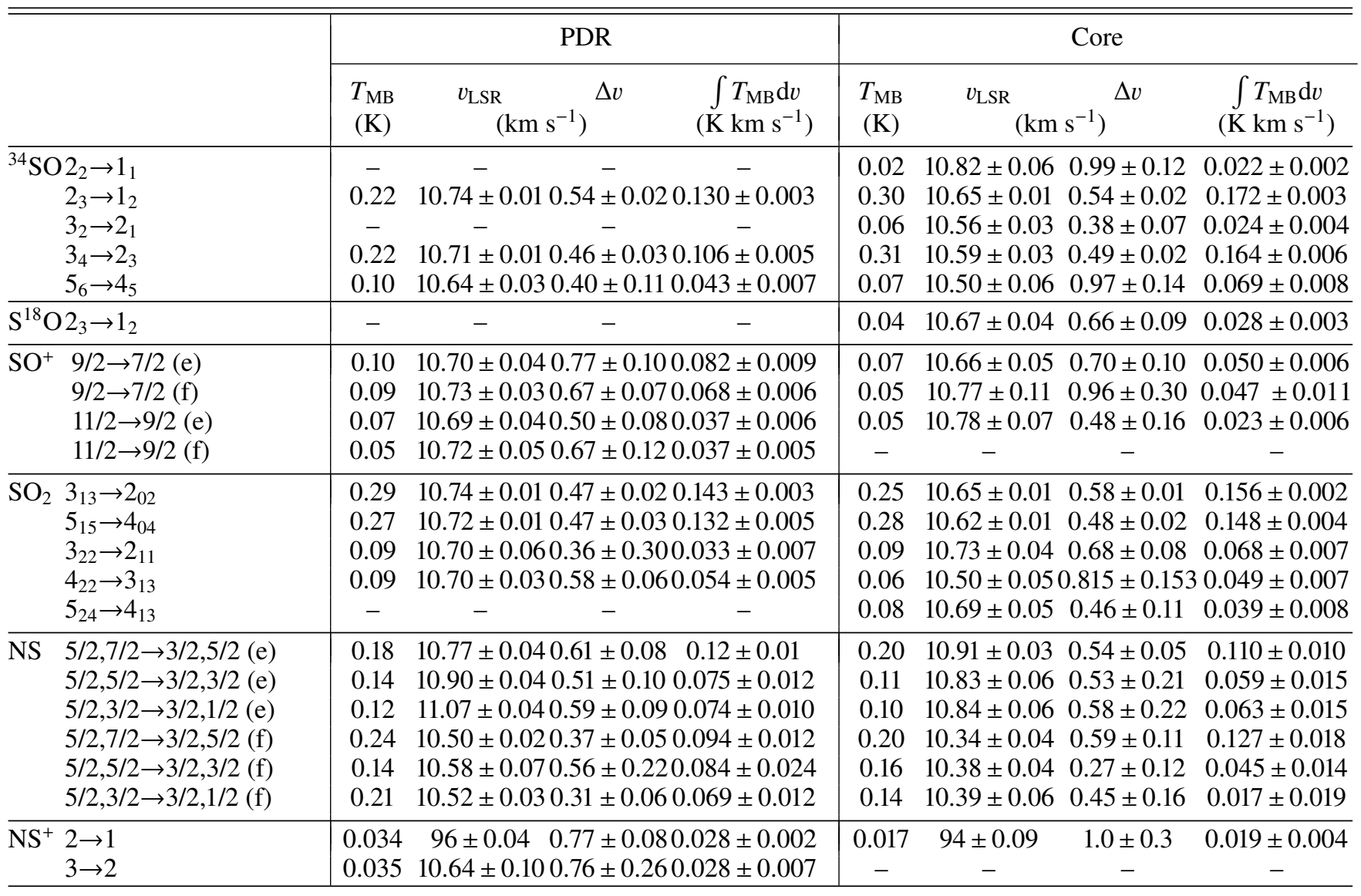



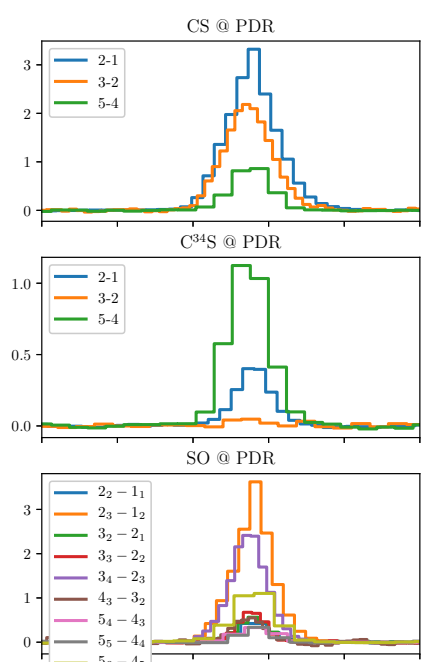

— $5 _ { 6 } - 4 _ { 5 } \longdiv { \mathrm { S } ^ { 1 8 } \mathrm { O } @ \mathrm { PDR } }$
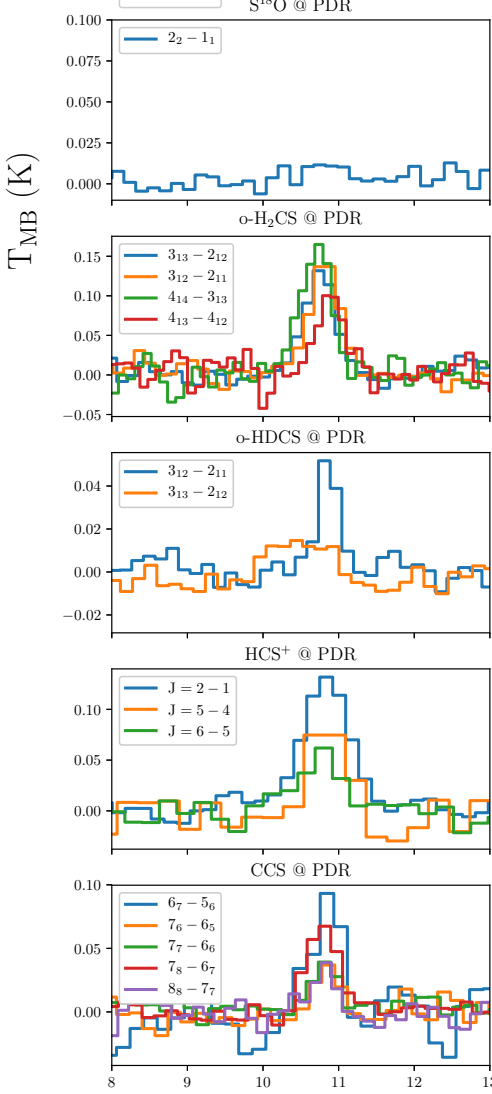
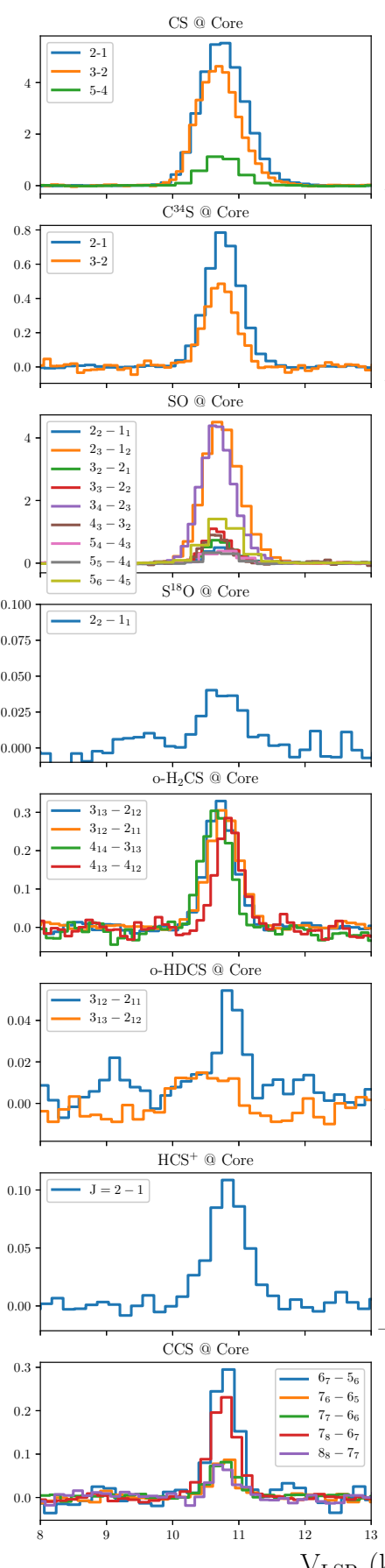
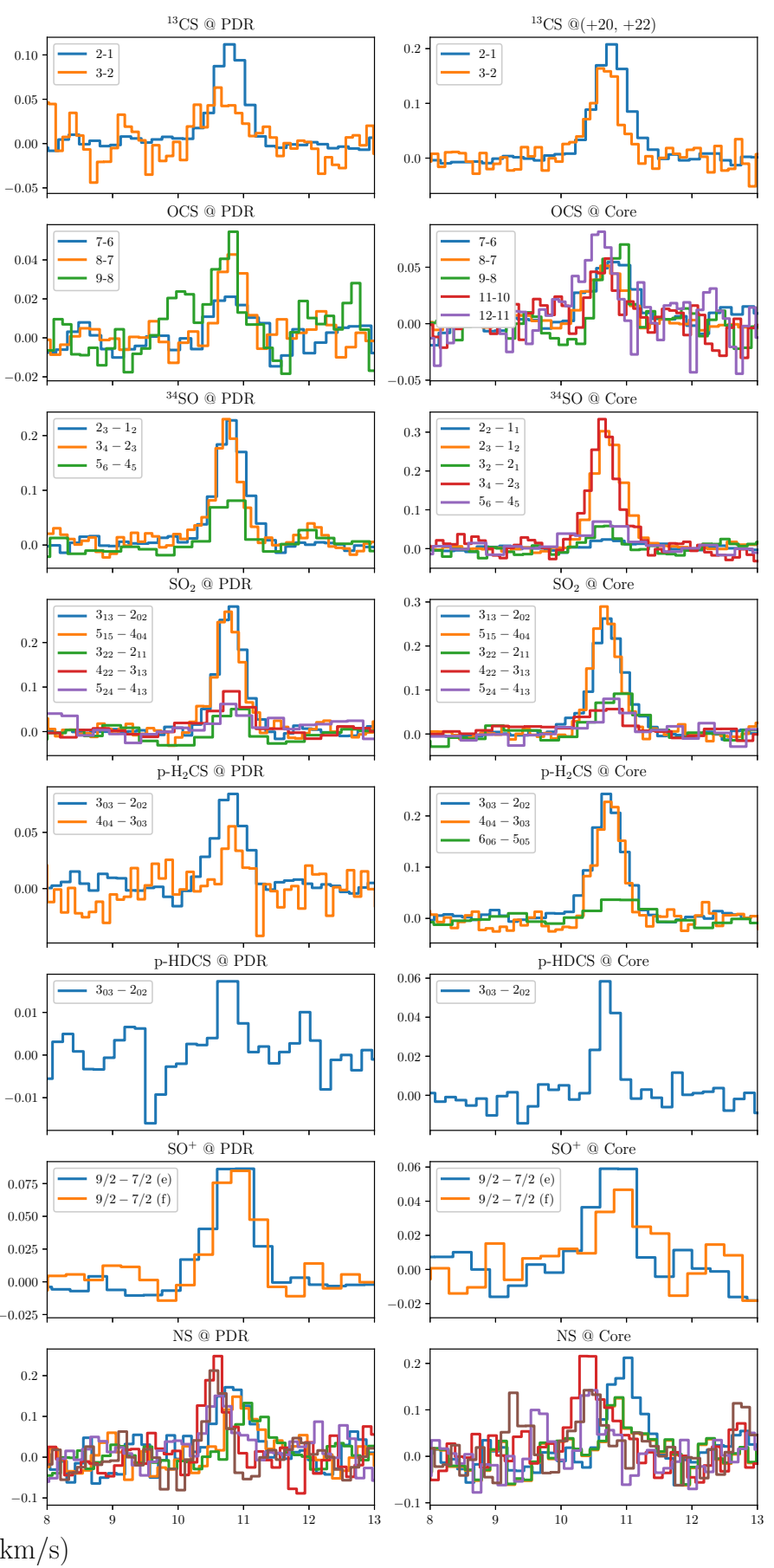

Fig. A.1. Detected species in the Horsehead. 


\section{Appendix B: Distribution of model parameters from MCMC simulations}
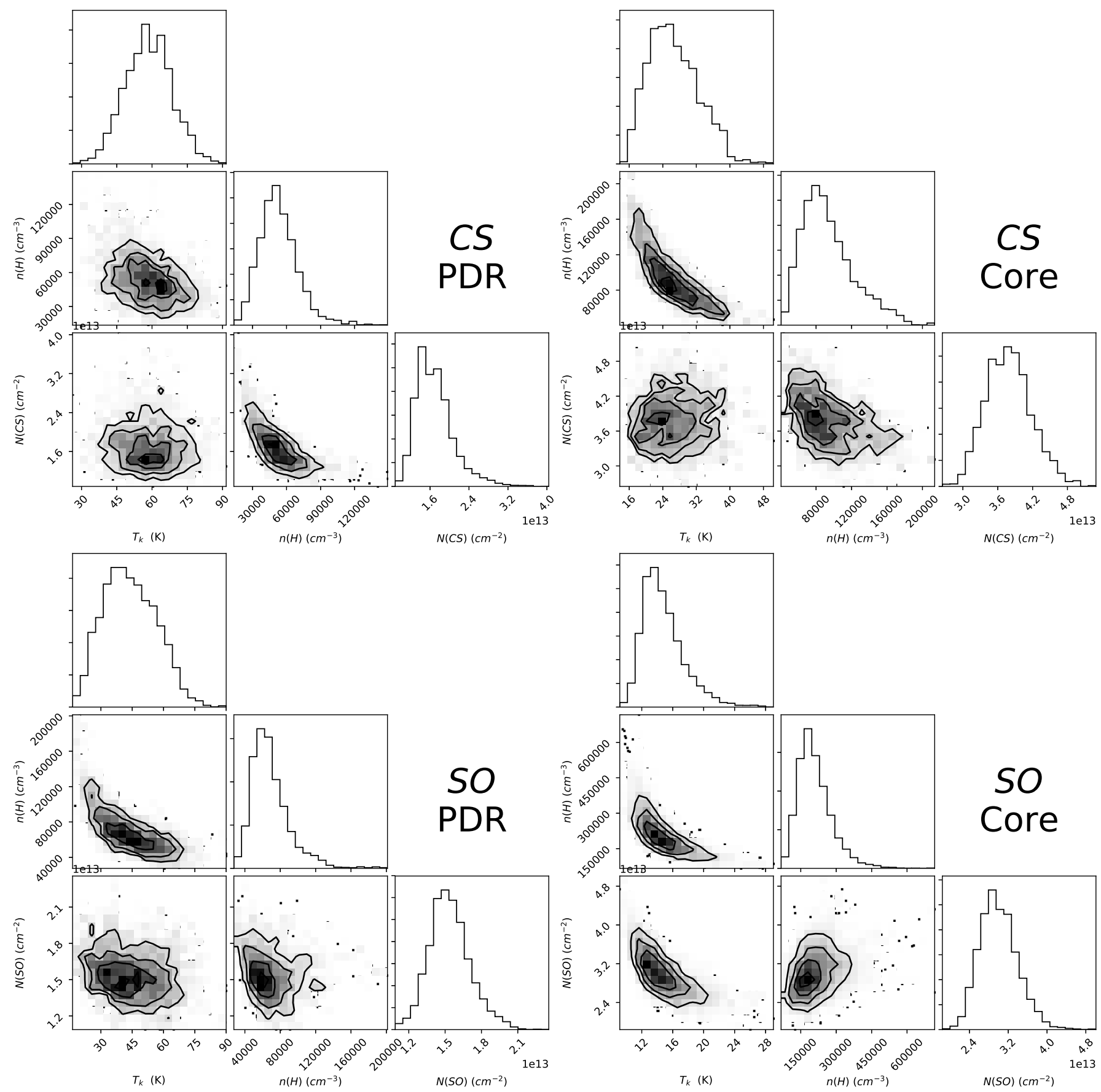

Fig. B.1. Histograms of the MCMC RADEX parameter distribution for the different molecules and regions studied. 
A\&A 628, A16 (2019)
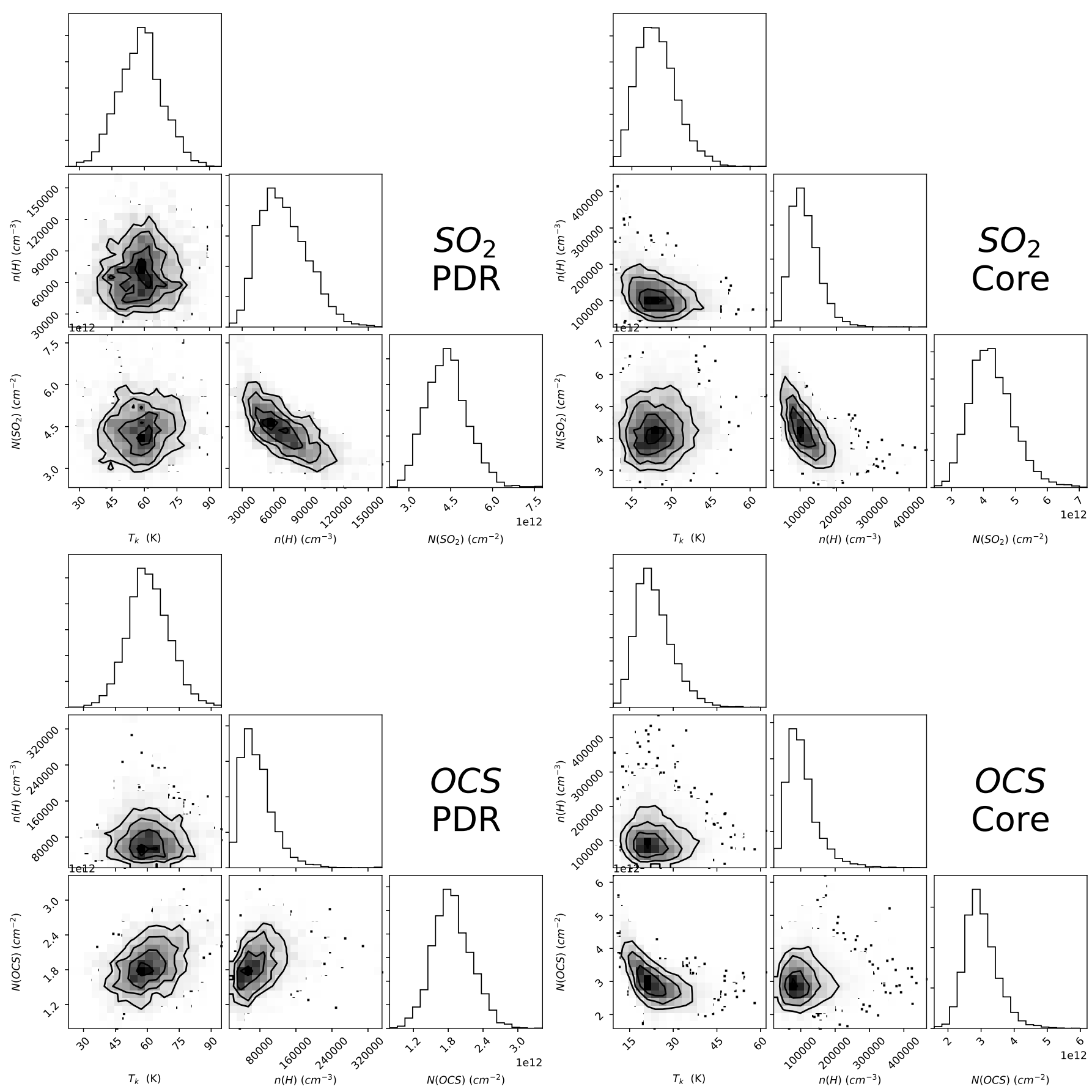

Fig. B.1. continued. 
P. Rivière-Marichalar et al.: Abundances of sulphur molecules in the Horsehead nebula
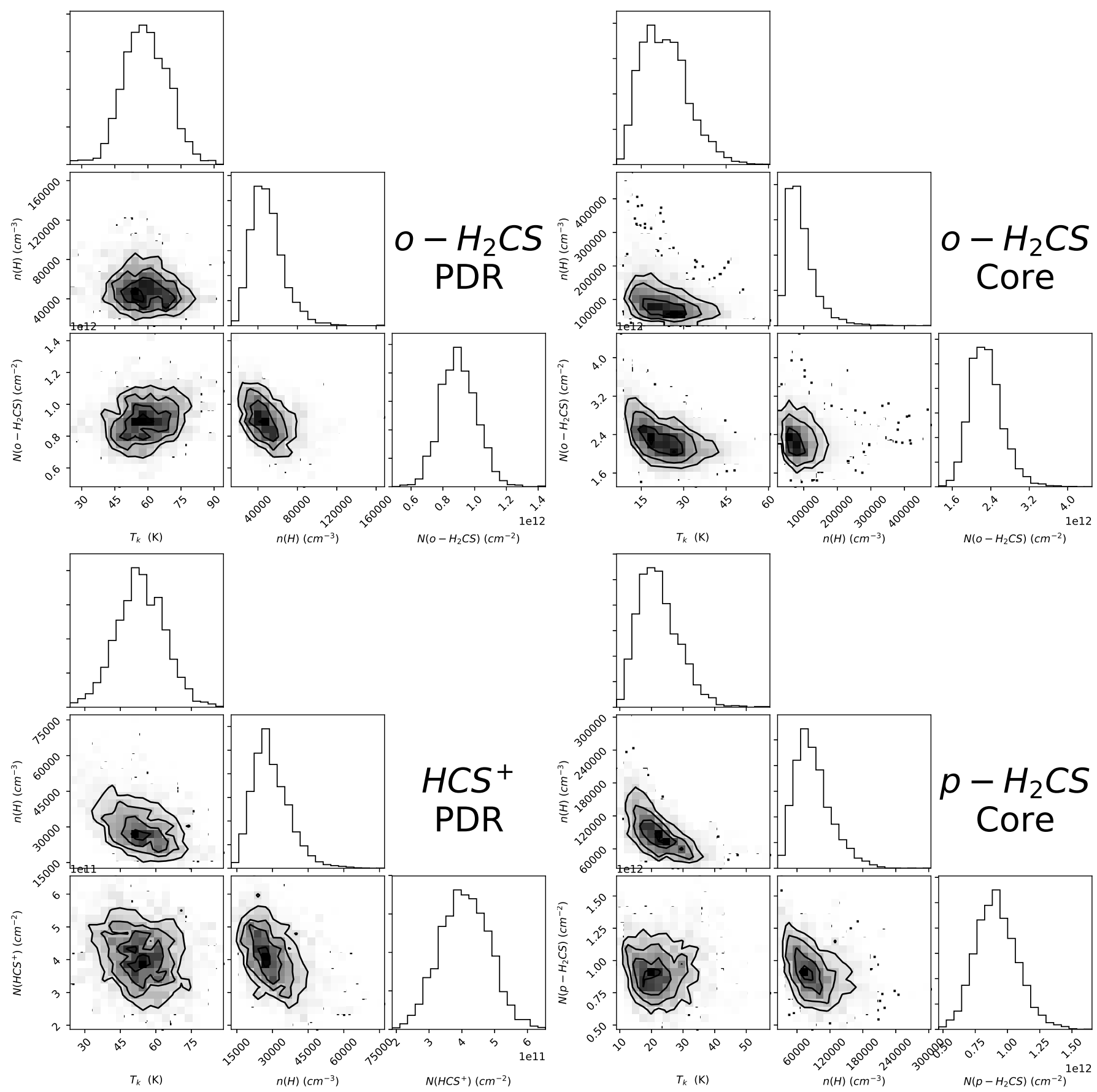

Fig. B.1. continued. 